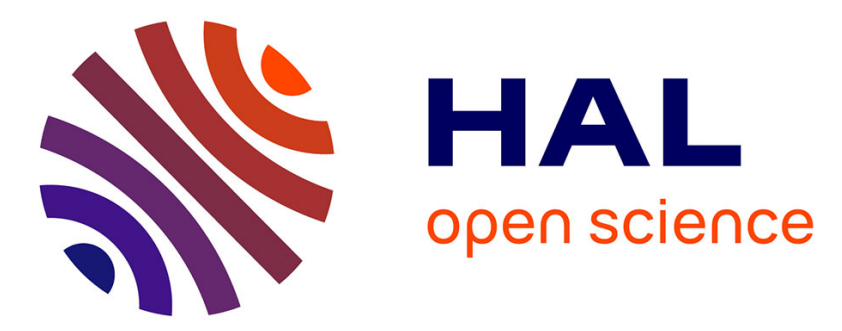

\title{
Bending analysis of composite laminated and sandwich structures using sublaminate variable-kinematic Ritz models
}

\author{
M. d'Ottavio, L. Dozio, R. Vescovini, O. Polit
}

\section{- To cite this version:}

M. d'Ottavio, L. Dozio, R. Vescovini, O. Polit. Bending analysis of composite laminated and sandwich structures using sublaminate variable-kinematic Ritz models. Composite Structures, 2016, 155, pp.4562. 10.1016/j.compstruct.2016.07.036 . hal-01367032

\section{HAL Id: hal-01367032 \\ https://hal.science/hal-01367032}

Submitted on 9 Jan 2019

HAL is a multi-disciplinary open access archive for the deposit and dissemination of scientific research documents, whether they are published or not. The documents may come from teaching and research institutions in France or abroad, or from public or private research centers.
L'archive ouverte pluridisciplinaire HAL, est destinée au dépôt et à la diffusion de documents scientifiques de niveau recherche, publiés ou non, émanant des établissements d'enseignement et de recherche français ou étrangers, des laboratoires publics ou privés. 


\title{
Bending analysis of composite laminated and sandwich structures using sublaminate variable-kinematic Ritz models
}

\author{
M. D’Ottavio, L. Dozio, R. Vescovini, O. Polit
}

\section{Introduction}

The ability to accurately predict complex stress distributions, both in terms of normal and transverse stress components, is a crucial aspect to assist the design of modern composite structures. Particularly relevant is the development of analysis tools that can efficiently combine the accuracy of the predictions with a reasonable computational effort, so that the effect of different stacking sequences, material properties or structural configurations, can be assessed since the preliminary design steps.

Several modeling strategies have been developed in the years, including Equivalent Single Layer (ESL) and Layer-Wise (LW) theories [1]. In ESL models, the displacement field is approximated starting from the description of one single reference surface; the number of degrees of freedom is thus independent of the number of layers. However, the in-plane displacement components are approximated with functions that are, at least, $C^{1}$ continuous along the thickness, which can be unable to capture the kinematics between layers with drastically different mechanical properties.

\footnotetext{
* Corresponding author.

E-mail address: michele.d_ottavio@u-paris10.fr (M. D’Ottavio).
}

One approach aiming at overcoming this restriction consists in the adoption of Zig-Zag functions, whose idea is to enrich the kinematics of ESL models by means of shape functions with discontinuous slope at the interface between layers. A comprehensive review of Zig-Zag theories, which is not the subject of the present work, is provided in [2].

Layerwise models are another class of theories directed toward the possibility of capturing complex responses along the throughthe-thickness direction. In this case, the displacement field is $C^{0}$ continuous along the thickness direction and is described with independent degrees of freedom for each of the plies composing the laminate. It follows that abrupt changes of strains between the layers can be properly detected. However, the number of theory-related degrees of freedom can be high, in particular for multilayered structures composed of a large number of plies.

Observing that the most important variations of elastic properties are generally confined to a subset of layer interfaces - for instance, between the core and the facesheet in the case of a sandwich - an effective idea is to group the plies into smaller sets, sometimes denoted as sublaminates, sharing the same kinematic description. An example is found in [3], where sandwich beams are analyzed using beam theory for the skins and a 
two-dimensional elasticity theory for the core. Refined plate and beam theories where the laminate is represented as an assemblage of sublaminates are discussed in [4-6]. In these formulations, firstorder zig-zag kinematics are postulated within each sublaminate. It follows that the in-plane displacement components vary in a piecewise fashion along the thickness, while the normal displacement component varies linearly. The extension to high-order zig-zag kinematics is discussed in [7]. Another approach based on the sublaminate description is found in $[8,9]$, where the displacement field is expanded with a third-order theory, and shear warping and shear coupling functions are used to ensure continuity of in-plane displacements, inter-laminar shear stresses and transverse normal stresses. An idealization of the sandwich into three sublaminates, each of them modeled with FSDT, is proposed in Ref. [10] with regard to the finite element implementation.

While the idea of sublaminate has been adopted by several authors, the formulations in the literature are commonly developed for specific kinematic theories. A theoretical framework that employs a general sublaminate approach in conjunction with general kinematics assumptions has been presented in [11], where a strong form analysis of the governing equations has been employed. The development of a sublaminate model in the context of a variationally consistent variable-kinematic approach has been recently performed by one of the authors [12]: the sublaminate description is coupled with a variable-kinematic theory expressed in a unified formulation, and the governing equations are obtained through the use of displacement-based or mixed variational statements. A vast amount of literature is available regarding unified theories $[13,14]$ and their implementation in the context of numerical procedures based on the finite element method [15-19], radial basis functions [20], quadrature techniques [21,22] and Ritz method [23-25], or of exact solutions based on Navier [26-28] and Levy methods [29-31]. The original idea of unified formulation - due to Carrera and often referred to as CUF (Carrera's Unified Formulation) -, offers the advantage of providing a systematic approach for developing formulations based on ESL and LW theories, of various order, in the context of the same framework. An interesting extension of CUF is due to Demasi [32-37] and is represented by the so-called Generalized Unified Formulation (GUF), the main distinction with CUF being the possibility of expanding each displacement field component by means of different theories and different orders. In the work of Ref. [12], the subdivision of the laminate into sublaminates has been proposed in conjunction with GUF thus leading to the Sublaminate-GUF (S-GUF) approach, and benchmark results are derived from the Navier solutions of the strong-form governing equations. In the present paper, the implementation of the S-GUF approach is discussed in the context of a displacement-based formulation, where the Ritz method is employed as solution technique. The main advantage of the Ritz approach consists in permitting the analysis of any combination of boundary conditions. Furthermore, no restrictions on the stacking sequences exist, so that realistic configurations characterized by the presence of membrane and/or flexural anisotropy can be accounted for. An overview of the S-GUF theoretical framework is provided in Section 2, while a description of the approximate solution approach based on the method of Ritz, together with the assembly and the expansion of the governing equations, is discussed in Section 3. A comprehensive set of test cases is discussed in Section 4, where results from literature are taken for validation purposes and novel benchmark results proposed.

\section{The Sublaminate Generalized Unified Formulation}

The formulation here presented provides a unified and versatile framework capable of generating multiple-kinematic models of increasing complexity (from classical FSDT models to higherorder full LW models) such that the desired balance of accuracy and computational cost can be obtained for the solution of a wide range of multilayered plate problems. This goal is achieved through the concept of selective ply grouping, or sublaminate, and the variable-kinematic capabilities of the generalized unified formulation (GUF) [32,33]. For this reason, the theoretical framework here proposed will be denoted as Sublaminate Generalized Unified Formulation (S-GUF). The fundamental element of S-GUF is the sublaminate, which is defined as a specific group of adjacent material plies with a specific 2D kinematic description, i.e., the theory adopted to approximate the displacement field across the thickness of the sublaminate. Accordingly, each sublaminate is associated with the following parameters: the number of plies of the sublaminate, the first and last ply constituting the sublaminate, and the local kinematic description, i.e. the Equivalent Single Layer (ESL) or Layerwise (LW) model to approximate the displacement field within the sublaminate. It is important to remark that plate descriptions combining both ESL and LW theories can be accounted for. For instance, a group of plies belonging to a sublaminate could be represented with a ESL description, while those belonging to another sublaminate could be modeled in a LW manner. Similarly, the order of the theory can be chosen independently from sublaminate to sublaminate. One example could be represented by a sandwich panel, whose facesheets are modeled with a low-order ESL theory, while a higher-order theory is adopted for the core. When the laminate is modeled by using one single sublaminate, the classical ESL and LW models are directly recovered.

\subsection{Geometric description}

The idealization of the multilayered structure as an assembly of perfectly bonded physical plies and mathematical sublaminates is illustrated in Fig. 1. As seen, the laminate is composed of $N_{p}$ plies of homogeneous, orthotropic material, that are numbered from the bottom to the top of the panel. The thickness of each single ply is denoted as $h_{p}$, so that the total thickness of the laminate is $h=\sum_{p=1}^{N_{p}} h_{p}$. Following the S-GUF approach, the laminate is subdivided into $k=1,2, \ldots, N_{k}$ sublaminates, numbered from the bottom to the top, each of them characterized by thickness $h_{k}$.

The number of plies of the $k$ th sublaminate is denoted as $N_{p}^{k}$, thus $\sum_{k=1}^{N_{k}} N_{p}^{k}=N_{p}$. A local numbering of plies $p=1, \ldots, N_{p}^{k}$ is also introduced at sublaminate level (see Fig. 2), where the first ply of the sublaminate is $p=1$, and the last ply is $N_{p}^{k}$. Accordingly, all the relevant quantities belonging to ply $p$ of sublaminate $k$ will be, hereinafter, explicitly indicated with the superscript ()$^{p, k}$.

Note that $z \in[-h / 2, h / 2]$ defines the global thickness coordinate, whereas $z_{p} \in\left[-h_{p} / 2, h_{p} / 2\right]$ and $z_{k} \in\left[-h_{k} / 2, h_{k} / 2\right]$ are the local ply and sublaminate coordinates, respectively. Corresponding nondimensional coordinates are introduced as

$\zeta_{p}=\frac{z_{p}}{h_{p} / 2} \quad$ and $\quad \zeta_{k}=\frac{z_{k}}{h_{k} / 2}$

and are linked through the following relation:

$\zeta_{p}=\frac{h_{k}}{h_{p}} \zeta_{k}+\frac{2}{h_{p}}\left(z_{0 k}-z_{0 p}\right)$

where $z_{0 p}$ and $z_{0 k}$ are the midplane coordinates of the $p$ th ply and $k$ th laminate, respectively.

\subsection{Kinematic approximation at sublaminate level}

In the context of the S-GUF formulation, each sublaminate is associated with a specific kinematic assumption that is defined 


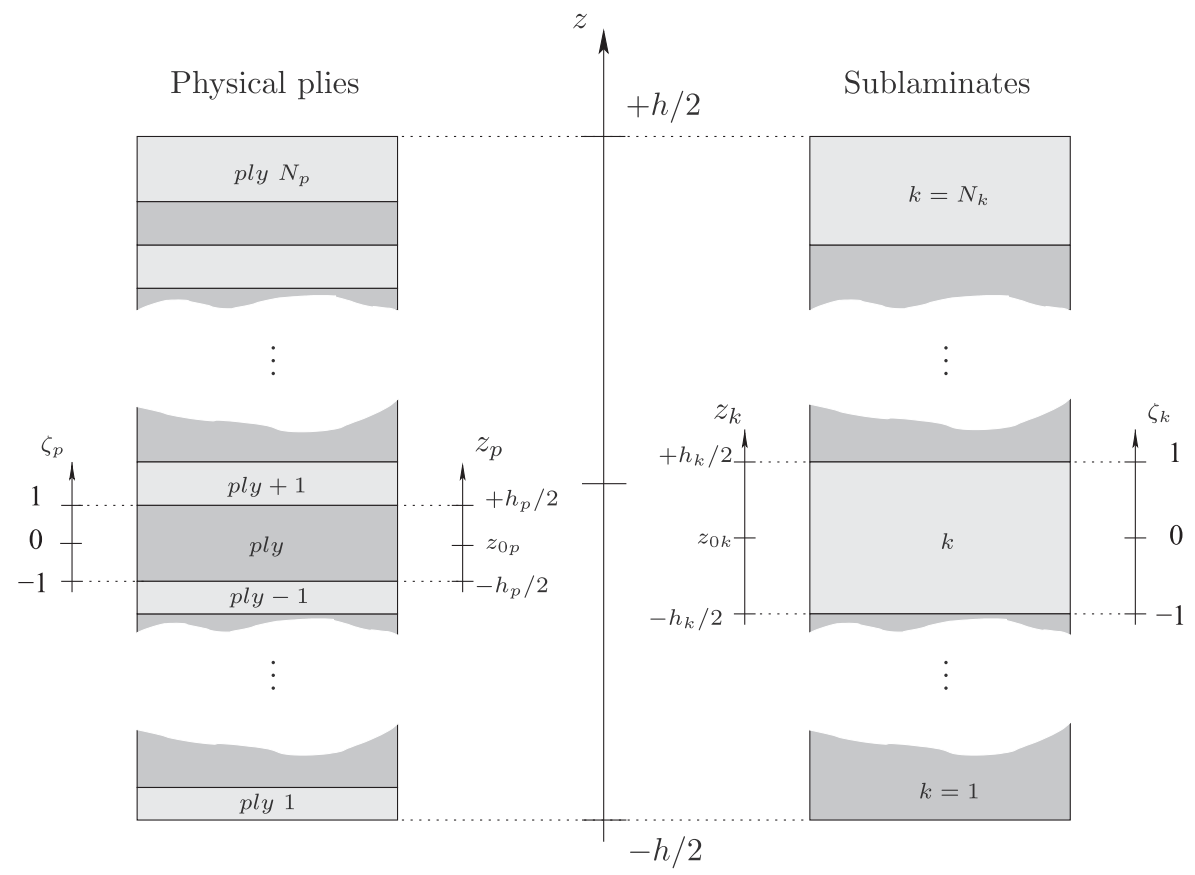

Fig. 1. S-GUF: geometric description.

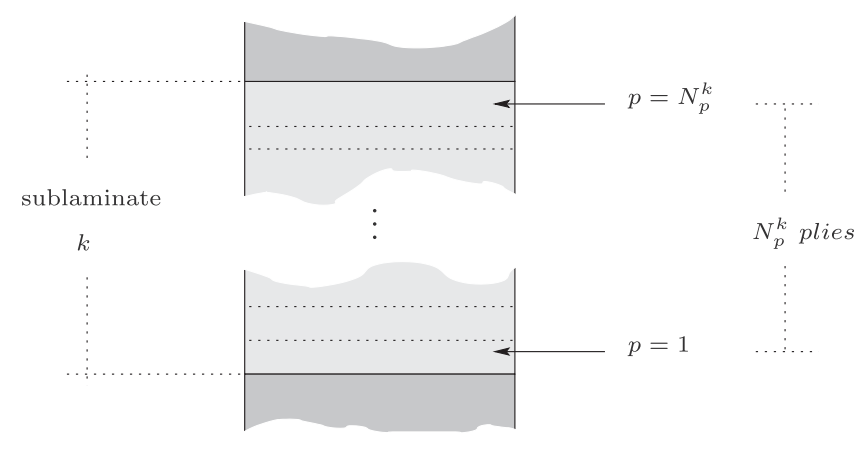

Fig. 2. First and last physical ply inside the sublaminate $k$.

in terms of theory and order of the expansion. The displacement field associated with the generic ply $p$ of the sublaminate $k$ is denoted as:

$\mathbf{u}^{p, k}=\left\{\begin{array}{lll}u_{x}^{p, k} & u_{y}^{p, k} & u_{z}^{p, k}\end{array}\right\}^{\mathrm{T}}$

Following the compact notation of GUF, the components of the displacement field defined by Eq. (3) are approximated as:

$$
\begin{cases}u_{x}^{p, k}\left(x, y, z_{p}\right)=F_{\alpha_{u_{x}}}\left(z_{p}\right) u_{x \alpha_{u_{x}}}^{p, k}(x, y) & \alpha_{u_{x}}=0,1, \ldots, N_{u_{x}}^{k} \\ u_{y}^{p, k}\left(x, y, z_{p}\right)=F_{\alpha_{u_{y}}}\left(z_{p}\right) u_{y \alpha_{u_{y}}}^{p, k}(x, y) & \alpha_{u_{y}}=0,1, \ldots, N_{u_{y}}^{k} \\ u_{z}^{p, k}\left(x, y, z_{p}\right)=F_{\alpha_{u_{z}}}\left(z_{p}\right) u_{z \alpha_{u_{z}}}^{p, k}(x, y) & \alpha_{u_{z}}=0,1, \ldots, N_{u_{z}}^{k}\end{cases}
$$

where the summation is implied over the repeated indexes $\alpha_{u_{x}}, \alpha_{u_{y}}$ and $\alpha_{u_{z}}$. The terms $F_{\alpha_{u_{r}}}$ define the thickness functions and approximate the displacement field along the normal-to-the-plane direction, while $u_{i \alpha_{u_{r}}}^{p, k}$ are the kinematic variables of the 2D approximation of the generic ply $p$ of the sublaminate $k$.

The theory-related degrees of freedom associated with each sublaminate are then given by $\left(N_{u_{x}}^{k}+1\right)+\left(N_{u_{y}}^{k}+1\right)+\left(N_{u_{z}}^{k}+1\right)$. It is important to highlight that both the theory and the order of the expansion can be, in general, different from sublaminate to sublaminate. On the other hand, the S-GUF is developed under the assumption that all the plies belonging to the same sublaminate are approximated with the same theory and the same order. It can be noted that the description of Eq. (4) covers both ESL and LW kinematic descriptions of the sublaminate. Indeed, a LW representation of the $k$ th sublaminate made of $N_{p}^{k}$ physical plies can be reduced to an ESL model by setting $z_{p}=z_{k}$ and

$$
\begin{aligned}
& u_{r}^{p, k}=u_{r}^{k} \\
& u_{r \alpha_{u_{r}}}^{p, k}=u_{r \alpha_{u_{r}}}^{k}
\end{aligned} \quad \forall p \in\left[1, N_{p}^{k}\right] \quad(r=x, y, z)
$$

where the index $r$ denotes the generic coordinate $x, y$ or $z$. From Eq. (5), it can be seen that an ESL model can be considered as a particular case of a LW model where the displacement unknowns of different plies are forced to be the same.

The thickness functions of Eq. (4) are expressed in terms of the non-dimensional coordinate $\zeta$ as:

$$
\begin{aligned}
\text { if } N_{u_{r}}^{k}=0: & F_{0}(\zeta)=1 \\
\text { if } N_{u_{r}}^{k}>0: & F_{0}(\zeta)=\frac{1+\zeta}{2} \\
& F_{1}(\zeta)=\frac{1-\zeta}{2} \\
& F_{l}(\zeta)=P_{l}(\zeta)-P_{l-2}(\zeta) \quad l=2,3, \ldots, N_{u_{r}}^{k}
\end{aligned}
$$

where $\zeta=\zeta_{k}$ for an ESL description, and $\zeta=\zeta_{p}$ for a LW description. The term $P_{l}(\zeta)$ is the Legendre's polynomial of order $l$ which is defined recursively as:

$P_{0}=1 ; \quad P_{1}=\zeta ; \quad P_{l+1}=\frac{(2 l+1) \zeta P_{l}-l P_{l-1}}{l+1}$

It is worth noting that, for $\zeta=1$, all the thickness functions $F_{\alpha}$ are equal to zero except $F_{0}$, i.e.,

at $\zeta=1 \quad\left\{\begin{array}{l}F_{0}=1 \\ F_{\alpha}=0 \quad \alpha=1, \ldots, N\end{array}\right.$

Furthermore, when $\zeta=-1$, all $F_{\alpha}=0$ except for $F_{1}$, i.e.,

at $\zeta=-1 \quad\left\{\begin{array}{l}F_{1}=1 \\ F_{\alpha}=0 \quad \alpha=0,2, \ldots, N\end{array}\right.$ 
The above properties imply that, for each displacement component $(r=x, y, z)$, the displacement at the top and the bottom is equal to one single kinematic variable, i.e., for an ESL description of the $k$ th sublaminate:

$u_{r}^{k}\left(\zeta_{k}=+1\right)=u_{r}^{k-\text { top }}=u_{r 0}^{k}$

$u_{r}^{k}\left(\zeta_{k}=-1\right)=u_{r}^{k-\text { bot }}=u_{r 1}^{k}$

and for an LW description of the $k$ th sublaminate:

$u_{r}^{p, k}\left(\zeta_{p}=+1\right)=u_{r}^{p, k-\text { top }}=u_{r 0}^{p, k}$

$u_{r}^{p, k}\left(\zeta_{p}=-1\right)=u_{r}^{p, k-\text { bot }}=u_{r 1}^{p, k}$

where 'top' and 'bot' denote the top and bottom face of the sublaminate or the ply where the displacement is defined. In other words, the kinematic variables associated with the expansion indexes $\alpha=0,1$ identify the displacement at the top and the bottom of the sublaminate or ply, respectively. This property is particularly useful during the assembly procedure since the continuity of the displacement field between adjacent plies or sublaminates can be easily imposed.

\subsection{Strain-displacement relation and Hooke's law}

Based on the assumption of infinitesimal displacements, the relation between strains and displacements reads:

$\varepsilon_{\Omega}^{p, k}=\mathbf{D}_{\Omega} \mathbf{u}^{p, k} \quad \varepsilon_{\mathrm{n}}^{p, k}=\mathbf{D}_{\mathrm{n}} \mathbf{u}^{p, k}+\mathbf{D}_{z} \mathbf{u}^{p, k}$

where, using the standard approach of CUF, the in-plane and the normal components are split into two vectors as:

$\varepsilon_{\Omega}^{p, k}=\left\{\begin{array}{lll}\varepsilon_{x x}^{p, k} & \varepsilon_{y y}^{p, k} & \gamma_{x y}^{p, k}\end{array}\right\}^{\mathrm{T}} ; \quad \varepsilon_{\mathrm{n}}^{p, k}=\left\{\begin{array}{lll}\gamma_{y z}^{p, k} & \gamma_{x z}^{p, k} & \varepsilon_{z z}^{p, k}\end{array}\right\}^{\mathrm{T}}$

and the differential matrices of Eq. (12) are:

$\mathbf{D}_{\Omega}=\left[\begin{array}{ccc}\frac{\partial}{\partial x} & 0 & 0 \\ 0 & \frac{\partial}{\partial y} & 0 \\ \frac{\partial}{\partial y} & \frac{\partial}{\partial x} & 0\end{array}\right] ; \quad \mathbf{D}_{\mathrm{n}}=\left[\begin{array}{ccc}0 & 0 & \frac{\partial}{\partial x} \\ 0 & 0 & \frac{\partial}{\partial y} \\ 0 & 0 & 0\end{array}\right] ; \quad \mathbf{D}_{z}=\left[\begin{array}{ccc}\frac{\partial}{\partial z} & 0 & 0 \\ 0 & \frac{\partial}{\partial z} & 0 \\ 0 & 0 & \frac{\partial}{\partial z}\end{array}\right]$

By adopting the same partitioning into in-plane and normal components of Eq. (12) and assuming linear orthotropic behaviour, the constitutive 3D law of the generic ply $p$ is written as:

$\boldsymbol{\sigma}_{\Omega}^{p, k}=\tilde{\mathbf{C}}_{\Omega \Omega}^{p, k} \varepsilon_{\Omega}^{p, k}+\tilde{\mathbf{C}}_{\Omega \mathrm{n}}^{p, k} \varepsilon_{\mathrm{n}}^{p, k} ; \quad \boldsymbol{\sigma}_{\mathrm{n}}^{p, k}=\tilde{\mathbf{C}}_{\mathrm{n} \Omega}^{p, k} \varepsilon_{\Omega}^{p, k}+\tilde{\mathbf{C}}_{\mathrm{nn}}^{p, k} \varepsilon_{\mathrm{n}}^{p, k}$

where

$\tilde{\mathbf{C}}_{\Omega \Omega}^{p, k}=\left[\begin{array}{ccc}\widetilde{C}_{11}^{p, k} & \widetilde{C}_{12}^{p, k} & \widetilde{C}_{16}^{p, k} \\ \widetilde{C}_{12}^{p, k} & \widetilde{C}_{22}^{p, k} & \widetilde{C}_{26}^{p, k} \\ \widetilde{C}_{16}^{p, k} & \widetilde{C}_{26}^{p, k} & \widetilde{C}_{66}^{p, k}\end{array}\right] ; \quad \tilde{\mathbf{C}}_{\Omega \mathrm{n}}^{p, k}=\left[\begin{array}{ccc}0 & 0 & \widetilde{C}_{13}^{p, k} \\ 0 & 0 & \widetilde{C}_{23}^{p, k} \\ 0 & 0 & \widetilde{C}_{36}^{p, k}\end{array}\right] ;$

$\tilde{\mathbf{C}}_{\mathrm{nn}}^{p, k}=\left[\begin{array}{ccc}\widetilde{C}_{44}^{p, k} & \widetilde{C}_{45}^{p, k} & 0 \\ \widetilde{C}_{45}^{p, k} & \widetilde{C}_{55}^{p, k} & 0 \\ 0 & 0 & \widetilde{C}_{33}^{p, k}\end{array}\right]$

\subsection{Variational formulation}

The formulation is developed within a variational displacement-based approach. More specifically, the weak form of the equilibrium equations is here expressed by means of the Principle of Virtual Displacements (PVD), which is written as:

$\int_{\Omega} \int_{-h / 2}^{h / 2} \delta \boldsymbol{\varepsilon}^{\mathrm{T}} \boldsymbol{\sigma} \mathrm{d} z \mathrm{~d} \Omega=\int_{\Omega} \delta u_{z}^{\text {top }} f_{z}^{\mathrm{top}} \mathrm{d} \Omega+\int_{\Omega} \delta u_{z}^{\text {bot }} f_{z}^{\text {bot }} \mathrm{d} \Omega$ where $\Omega$ is the reference surface, $\boldsymbol{\sigma}$ and $\boldsymbol{\varepsilon}$ are the vectors collecting the stress and strain components, respectively, and the superscripts 'top' and 'bot' denote quantities referred to the top and the bottom of the laminate. The right-hand side of Eq. (17) is the external virtual work due to a set of normal pressure loads applied at the top and the bottom of the laminate. For simplicity the applied load is here restricted to the case of a normal pressure, but the formulation can be easily extended to account for more general loading, including forces per unit length or volume, as well as concentrated loads.

According to the subdivision of the plate into $N_{k}$ sublaminates, each one including $N_{p}^{k}$ physical plies with local kinematic field $\mathbf{u}^{p, k}$, stress field $\boldsymbol{\sigma}^{p, k}$ and strain field $\boldsymbol{\varepsilon}^{p, k}$, the PVD can be written in the following form

$$
\begin{gathered}
\sum_{k=1}^{N_{k}} \sum_{p=1}^{N_{p}^{k}} \int_{\Omega} \int_{z_{p}^{\mathrm{bot}}}^{z_{p}^{\mathrm{top}}}\left(\delta \boldsymbol{\varepsilon}_{\Omega}^{p, k^{\mathrm{T}}} \boldsymbol{\sigma}_{\Omega}^{p, k}+\delta \boldsymbol{\varepsilon}_{\mathrm{n}}^{p, k^{\mathrm{T}}} \boldsymbol{\sigma}_{\mathrm{n}}^{p, k}\right) \mathrm{d} z \mathrm{~d} \Omega \\
=\int_{\Omega} \delta u_{z}^{\mathrm{top}} f_{z}^{\mathrm{top}} \mathrm{d} \Omega+\int_{\Omega} \delta u_{z}^{\mathrm{bot}} f_{z}^{\mathrm{bot}} \mathrm{d} \Omega
\end{gathered}
$$

After substitution of Eqs. (12) and (15) into Eq. (18), the two contributions to the internal virtual work are expressed as a function of the displacement variables as:

$$
\begin{aligned}
\delta \varepsilon_{\Omega}^{p, k^{\mathrm{T}}} \boldsymbol{\sigma}_{\Omega}^{p, k}= & \delta \boldsymbol{\varepsilon}_{\Omega}^{p, k^{\mathrm{T}}}\left(\tilde{\mathbf{C}}_{\Omega \Omega}^{p, k} \varepsilon_{\Omega}^{p, k}+\tilde{\mathbf{C}}_{\Omega \mathrm{n}}^{p, k} \varepsilon_{\mathrm{n}}^{p, k}\right) \\
= & \delta\left(\mathbf{D}_{\Omega} \mathbf{u}^{p, k}\right)^{\mathrm{T}} \tilde{\mathbf{C}}_{\Omega \Omega}^{p, k} \mathbf{D}_{\Omega} \mathbf{u}^{p, k}+\delta\left(\mathbf{D}_{\Omega} \mathbf{u}^{p, k}\right)^{\mathrm{T}} \tilde{\mathbf{C}}_{\Omega \mathrm{n}}^{p, k} \mathbf{D}_{\mathrm{n}} \mathbf{u}^{p, k} \\
& +\delta\left(\mathbf{D}_{\Omega} \mathbf{u}^{p, k}\right)^{\mathrm{T}} \tilde{\mathbf{C}}_{\Omega \mathrm{n}}^{p, k} \mathbf{D}_{z} \mathbf{u}^{p, k} \\
\delta \boldsymbol{\varepsilon}_{\mathrm{n}}^{p, k^{\mathrm{T}}} \boldsymbol{\sigma}_{\mathrm{n}}^{p, k}= & \delta\left(\mathbf{D}_{\mathrm{n}} \mathbf{u}^{p, k}+\mathbf{D}_{z} \mathbf{u}^{p, k}\right)^{\mathrm{T}}\left(\tilde{\mathbf{C}}_{\mathrm{n} \Omega}^{p, k} \varepsilon_{\Omega}^{p, k}+\tilde{\mathbf{C}}_{\mathrm{nn}}^{p, k} \varepsilon_{\mathrm{n}}^{p, k}\right) \\
= & \delta\left(\mathbf{D}_{\mathrm{n}} \mathbf{u}^{p, k}\right)^{\mathrm{T}} \tilde{\mathbf{C}}_{\mathrm{n} \Omega}^{p, k} \mathbf{D}_{\Omega} \mathbf{u}^{p, k}+\delta\left(\mathbf{D}_{\mathrm{n}} \mathbf{u}^{p, k}\right)^{\mathrm{T}} \tilde{\mathbf{C}}_{\mathrm{nn}}^{p, k} \mathbf{D}_{\mathrm{n}} \mathbf{u}^{p, k} \\
& +\delta\left(\mathbf{D}_{\mathrm{n}} \mathbf{u}^{p, k}\right)^{\mathrm{T}} \tilde{\mathbf{C}}_{\mathrm{nn}}^{p, k} \mathbf{D}_{z} \mathbf{u}^{p, k}+\delta\left(\mathbf{D}_{z} \mathbf{u}^{p, k}\right)^{\mathrm{T}} \tilde{\mathbf{C}}_{\mathrm{n} \Omega}^{p, k} \mathbf{D}_{\Omega} \mathbf{u}^{p, k} \\
& +\delta\left(\mathbf{D}_{z} \mathbf{u}^{p, k}\right)^{\mathrm{T}} \tilde{\mathbf{C}}_{\mathrm{nn}}^{p, k} \mathbf{D}_{\mathrm{n}} \mathbf{u}^{p, k}+\delta\left(\mathbf{D}_{z} \mathbf{u}^{p, k}\right)^{\mathrm{T}} \tilde{\mathbf{C}}_{\mathrm{nn}}^{p, k} \mathbf{D}_{z} \mathbf{u}^{p, k}
\end{aligned}
$$

The PVD can be finally expressed as function of the kinematic variables $u_{r \alpha_{u r}}^{p, k}$ by substitution of the expansion of Eq. (4) into Eqs. (19) and (20).

\subsection{Ritz approximation}

Once a specific plate theory is postulated with regard to the through-the-thickness direction, the original 3D problem is transformed into a 2D problem in the $x-y$ plane. It follows that the equations expressing the equilibrium are partial differential equations, and the unknowns of the problem are the generalized displacement components, which are functions of the $x$ and $y$ coordinates. An exact solution of the governing PDE can be hardly found for any set of boundary conditions and stacking sequences, thus an approximate solution based on the method of Ritz is here proposed. In particular, the $2 \mathrm{D}$ variables of the assumed kinematic model are expressed as follows

$$
\left\{\begin{array}{l}
u_{x \alpha_{u_{x}}}^{p, k}(x, y)=N_{u_{x} i}(x, y) u_{x \alpha_{u_{x} i} i}^{p, k} \\
u_{y \alpha_{u_{y}}}^{p, k}(x, y)=N_{u_{y} i}(x, y) u_{y \alpha_{u_{y}} i}^{p, k} \quad i=1,2, \ldots, M \\
u_{z \alpha_{u_{z}}}^{p, k}(x, y)=N_{u_{z} i}(x, y) u_{z \alpha_{u_{z} i}}^{p, k}
\end{array}\right.
$$

where $N_{u_{r} 1}, N_{u_{r} 2}, \ldots, N_{u_{r} M}$ is the complete set of global, admissible and linearly independent functions selected to represent each kinematic unknown related to the expansion of the generic displacement component $u_{r}$. Note also that the admissible functions have no dependency on the ply index $p$ or the sublaminate index $k$, i.e., the boundary conditions of the plate are considered to be homogeneous through the height of the same section, so the same functions are adopted in each ply/sublaminate for the in-plane solution of the 
problem. The combination of the Ritz approximation in Eq. (21) within the framework of the S-GUF will be denoted hereinafter as S-GUF-Ritz.

By referring to a computational domain $(\xi, \eta)$ of the plate, with $\xi \in[-1,1]$ and $\eta \in[-1,1]$, the $i$ th Ritz function is taken as

$N_{u_{r} i}(\xi, \eta)=\phi_{u_{r} m}(\xi) \psi_{u_{r} n}(\eta) \quad m=1, \ldots, R ; n=1, \ldots, S$

where $R$ and $S$ are the number of functions to approximate the displacement field along the direction $\xi$ and $\eta$, respectively, and the following arrangement is chosen for defining the ith Ritz function in terms of the $m$ th and $n$th one-dimensional functions:

$i=S(m-1)+n$

The one-dimensional functions $\phi_{u_{r} m}(\xi)$ and $\psi_{u_{r} n}(\eta)$ are expressed, in turn, as the product between a complete set of functions and proper boundary functions:

$\phi_{u_{r} m}(\xi)=f_{u_{r}}(\xi) p_{m}(\xi)$

$\psi_{u_{r} n}(\eta)=g_{u_{r}}(\eta) p_{n}(\eta)$

where the boundary functions are:

$f_{u_{r}}(\xi)=(1+\xi)^{e_{1 r}}(1-\xi)^{e_{2 r}}$

$g_{u_{r}}(\eta)=(1+\eta)^{e_{1 r}}(1-\eta)^{e_{2 r}}$

and the coefficients $e_{1 r}$ and $e_{2 r}$ can be either 0 or 1 and are chosen depending on the boundary conditions [23].

The functions $p_{m}$ and $p_{n}$ are here expressed using Legendre polynomials, so:

$p_{0}=1 ; \quad p_{1}=\zeta ; \quad p_{l+1}=\frac{(2 l+1) \zeta p_{l}-l p_{l-1}}{l+1} \quad(l=m, n)$

The expansion of Eq. (26) guarantees the completeness of the series, and is particularly advantageous thanks to the orthogonality properties of the Legendre polynomials.

\subsubsection{Principle of Virtual Displacements}

After substitution of Eqs. (4) and (22) into Eq. (18), the discrete expression of the Principle of Virtual Displacements is obtained as:

$\sum_{k=1}^{N_{k}} \sum_{p=1}^{N_{p}^{k}} \delta u_{r \alpha_{u_{r} i} i}^{p, k} \widetilde{C}_{R S}^{p, k} Z_{(\partial) u_{r}(\partial) u_{s}}^{p \alpha_{u_{r} \beta_{u_{s}}}} \mathcal{I}_{u_{r} u_{s} i j}^{\text {defg }} u_{s \beta u_{s} i}^{p, k}=\delta u_{z 0 i}^{N_{p}^{k}, N_{k}} \mathcal{I}_{u_{z} f_{z} i}^{\text {top }}+\delta u_{z 1 i}^{1,1} \mathcal{I}_{u_{z} f_{z} i}^{\text {bot }}$

where:

$\mathcal{I}_{u_{z} f_{z} i}^{\text {top }}=\int_{\Omega} N_{u_{z} i} f_{z}^{\text {top }}(x, y) \mathrm{d} \Omega ; \quad \mathcal{I}_{u_{z} f_{z} i}^{\text {bot }}=\int_{\Omega} N_{u_{z}} f_{z}^{\text {bot }}(x, y) \mathrm{d} \Omega$

The left-hand side of Eq. (27) is the internal virtual work, and the right-hand side is the virtual work due to the applied forces. For clarity purposes, the internal virtual work is here reported in a compact form. The full expression is provided in the Appendix A1. In the expression of Eq. (27), the term $\widetilde{C}_{R S}^{p, k}$ defines the generic $R S$ coefficient of the ply constitutive equation in the laminate reference system, while the term $Z_{(\partial) u_{r}(\partial) u_{s}}^{p \alpha_{u_{r}} \beta_{u_{s}}}$ specifies the generic integral of the thickness functions according to:

$$
\begin{aligned}
& Z_{u_{r} u_{s}}^{p \alpha_{u_{r}} \beta_{u_{s}}}=\int_{z_{p}^{\text {bot }}}^{z_{p}^{\text {top }}} F_{\alpha_{u_{r}}} F_{\beta_{u_{s}}} \mathrm{~d} z Z_{\partial u_{r} u_{s}}^{p \alpha_{u_{r}} \beta_{u_{s}}}=\int_{z_{p}^{\text {bot }}}^{z_{p}^{\text {top }}} F_{\alpha_{u_{r}}, z} F_{\beta_{u_{s}}} \mathrm{~d} z \\
& Z_{u_{r} \partial u_{s}}^{p \alpha_{u_{r}} \beta_{u_{s}}}=\int_{z_{p}^{\text {bot }}}^{z_{p}^{\text {top }}} F_{\alpha_{u_{r}}} F_{\beta_{u_{s}}, z} \mathrm{~d} z Z_{\partial u_{r} \partial u_{s}}^{p \alpha_{u_{r_{r}} \beta_{u_{s}}}}=\int_{z_{p}^{\mathrm{bot}}}^{z_{p}^{\text {top }}} F_{\alpha_{u_{r}}, z} F_{\beta_{u_{s}}, z} \mathrm{~d} z
\end{aligned}
$$

It can be noted that, according to Eq. (27), the expression of the internal virtual work is expressed by means of a self-repeating block, here denoted as kernel of the formulation, i.e., the term that is pre- and post-multiplied by the virtual and actual generalized displacement, respectively. As discussed next, the assembly of the governing equations is simply performed by properly expanding this kernel.

The Ritz integrals, denoted in Eq. (27) as $\mathcal{I}_{u_{r} u_{s} i j}^{\text {defg }}$, are defined as:

$\mathcal{I}_{u_{r} u_{s} i j}^{\text {defg }}=\int_{-1}^{1} \int_{-1}^{1} \frac{\partial^{d+e} N_{u_{r} i}}{\partial x^{d} \partial y^{e}} \frac{\partial^{f+g} N_{u_{s} j}}{\partial x^{f} \partial y^{g}} \mathrm{~d} \eta \mathrm{d} \xi \quad(d, e, f, g=0,1)$

It is here remarked that, for efficiency, all the integrals defined by Eq. (30) are calculated in a closed-form manner. The advantage of an analytical integration over a numerical one is twofold: firstly, the number of operations is much smaller; secondly, the resulting stiffness matrix is filtered from the presence of spurious not-null contributions, which has a beneficial effect on the sparsity of the matrix.

It is worth noting that in Eq. (27) the dependence on the thickness coordinate is confined to the term $Z_{(\partial) u_{r}(\partial) u_{s}}^{p \alpha_{u_{u}} \beta_{u_{s}}}$, while the dependence on the in-plane coordinates is part of the Ritz integrals

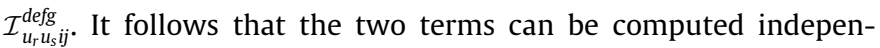
dently - thus reducing the number of nested summatories and the time needed to build-up the stiffness matrix - and their contribution is successively assembled.

\section{Expansion and assembly}

The discrete equations governing the equilibrium of the panel are obtained after expanding Eq. (27) over the theory-related indexes, and subsequently assembling the contributions at ply level first, and at sublaminate level next.

The theory expansion is performed by expanding the summations over the indexes $\alpha_{u_{r}}$ and $\beta_{u_{s}}$. In the context of the Generalized Unified Formulation, these two indexes can vary between $N_{r}^{k}$ and $N_{s}^{k}$ as far as the displacement components $u_{r}$ and $u_{s}$ can be, in principle, expanded up to a different order. By collecting the expanded terms into matrices and vectors, it is possible to re-write the PVD of Eq. (27) as:

$\sum_{k=1}^{N_{k}} \sum_{p=1}^{N_{p}^{k}} \delta \mathbf{u}_{r i}^{p, k^{\mathrm{T}}} \widetilde{C}_{R S}^{p, k} \mathbf{Z}_{(\partial) u_{r}(\partial) u_{s}}^{p, k} \mathcal{I}_{u_{r} u_{s} i j}^{\text {defg }} \mathbf{u}_{s j}^{p, k}=\delta u_{z 0 i}^{N_{p}^{k}, N_{k}} \mathcal{I}_{u_{z} f_{z} i}^{\text {top }}+\delta u_{z 1 i}^{1,1} \mathcal{I}_{u_{z} f_{z} i}^{\text {bot }}$

where the vectors $\mathbf{u}_{r i}^{p, k}$ and $\mathbf{u}_{s j}^{p, k}$ are those collecting the kinematic variables for each ply $p$ of the generic sublaminate $k$, and have dimensions $\left(N_{r}^{k}+1\right)$ and $\left(N_{s}^{k}+1\right)$, while the matrix of the thickness integrals, $\mathbf{Z}_{(\partial) u_{r}(\partial) u_{s}}^{p, k}$, has dimensions $\left(N_{r}^{k}+1\right) \times\left(N_{s}^{k}+1\right)$. To facilitate the assembly procedure and the imposition of the continuity of the displacements at ply interfaces, the vectors are organized such that the first entry corresponds to the displacement at the top, while the last entry is associated with the displacement at the bottom, viz.

$\mathbf{u}_{r i}^{p, k}=\left\{\begin{array}{lllll}u_{r 0 i}^{p, k} & u_{r 2 i}^{p, k} & \ldots & u_{r N_{u_{r} i}^{k} i}^{p, k} & u_{r 1 i}^{p, k}\end{array}\right\}^{\mathrm{T}}$

The second step of the assembly procedure is given by the summation over all plies constituting the $k$ th sublaminate, which is performed by cycling over the index $p$ of Eq. (31). The resulting expression of the PVD is then:

$\sum_{k=1}^{N_{k}} \delta \mathbf{u}_{r i}^{k^{T}} \mathbf{Z}_{(\partial) u_{r}(\partial) u_{s} R S}^{k} \mathcal{I}_{u_{r} u_{s} i j}^{\text {defg }} \mathbf{u}_{s j}^{k}=\delta u_{z 0 i}^{N_{p}^{k}, N_{k}} \mathcal{I}_{u_{z} f_{z} i}^{\text {top }}+\delta u_{z 1 i}^{1,1} \mathcal{I}_{u_{z} f_{z} i}^{\text {bot }}$

where the dependence on the elastic coefficients $\widetilde{C}_{R S}^{p, k}$ is now included in the thickness matrix, $\mathbf{Z}_{(\partial) u_{\mathrm{r}}(\partial) u_{s} R S}^{k}$, as highlighted by the indexes $R S$.

The way the assembly process is performed, and consequently the dimensions of the vectors and matrices of Eq. (33), depends on the theory that is adopted for the kinematic description of the 
Table 1

Assembly at sublaminate level: size of $\mathbf{Z}^{k}$ matrices.

\begin{tabular}{|c|c|c|}
\hline \multirow{2}{*}{$\begin{array}{l}\text { Kinematic description } \\
u_{r} / u_{s}\end{array}$} & \multicolumn{2}{|l|}{ Size of $\mathbf{Z}_{(\partial) u_{r}(\partial) u_{s} R S}^{k}$} \\
\hline & Rows $\left(N_{\mathrm{DOFu}_{\mathrm{r}}}^{k}\right)$ & Columns $\left(N_{\mathrm{DOF} u_{s}}^{k}\right)$ \\
\hline ESL/ESL & $N_{u_{r}}^{k}+1$ & $N_{u_{s}}^{k}+1$ \\
\hline ESL/LW & $N_{u_{r}}^{k}+1$ & $\left(N_{u_{s}}^{k}+1\right) N_{p}^{k}-\left(N_{p}^{k}-1\right)$ \\
\hline LW/ESL & $\left(N_{u_{r}}^{k}+1\right) N_{p}^{k}-\left(N_{p}^{k}-1\right)$ & $N_{u_{s}}^{k}+1$ \\
\hline LW/LW & $\left(N_{u_{r}}^{k}+1\right) N_{p}^{k}-\left(N_{p}^{k}-1\right)$ & $\left(N_{u_{s}}^{k}+1\right) N_{p}^{k}-\left(N_{p}^{k}-1\right)$ \\
\hline
\end{tabular}

sublaminate $k$. A sketch of the various possibilities is summarized in Table 1 , where the dimensions of the matrix $\mathbf{Z}_{(\partial) u_{r}(\partial) u_{s} R S}^{k}$ are reported depending on whether the displacement components $u_{r}$ and $u_{s}$ are represented with an ESL or a LW approach. In the first case, the vector collecting the unknowns of the sublaminate reads

$\mathbf{u}_{r i}^{k}=\mathbf{u}_{r i}^{p, k}=\mathbf{u}_{r i}^{p+1, k}$

meaning that the contributions associated with the various plies are directly superposed. In the case of a LW approach, the vector of the unknowns is organized as

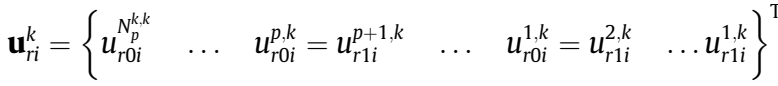

where the first and the last terms correspond to the displacement at the top- and the bottom-most plies of the sublaminate $k$. The intermediate entries of Eq. (35) express the $\left(N_{p}^{k}-1\right)$ continuity conditions between the various plies composing the sublaminate.

The third step of the assembly procedure is given by the expansion over the various sublaminates composing the laminate. Referring to the expression of Eq. (33), the PVD becomes:

$\delta \mathbf{u}_{r i}^{\mathrm{T}} \mathbf{Z}_{(\partial) u_{r}(\partial) u_{s} R s} \mathcal{I}_{u_{r} u_{s} i j}^{\text {defg }} \mathbf{u}_{s j}=\delta \mathbf{u}_{z i}^{\mathrm{T}} \mathbf{L}_{z i}^{\text {top }} \mathcal{I}_{u_{z} f_{z} i}^{\text {top }}+\delta \mathbf{u}_{z i}^{\mathrm{T}} \mathbf{L}_{z i}^{\text {bot }} \mathcal{I}_{u_{z} f_{z} i}^{\text {bot }}$

where

$\mathbf{u}_{r i}=\left\{\begin{array}{lll}\mathbf{u}_{r i}^{N_{k}} & \ldots & \mathbf{u}_{r i}^{1}\end{array}\right\}^{\mathrm{T}}$

and:

$\mathbf{L}_{z i}^{\text {top }}=\left\{\begin{array}{llll}1 & 0 & \ldots & 0\end{array}\right\}^{\mathrm{T}} ; \quad \mathbf{L}_{z i}^{\text {bot }}=\left\{\begin{array}{llll}0 & \ldots & 0 & 1\end{array}\right\}^{\mathrm{T}}$

where the two vectors of Eq. (38) are column-vectors of dimension $N_{\text {DOFu }}$. The assembly of the various sublaminates is performed in a LW manner. Since the displacement field along the thickness coordinate is approximated using Legendre polynomials irrespective of the ESL or LW description inside the sublaminates, the continuity between the displacement at the top of the sublaminate $k$ and the bottom of the sublaminate $k+1$ is easily imposed by enforcing the condition $u_{r 0 i}^{N_{p}^{k}, k}=u_{r 1 i}^{1, k+1}$.

Once the assembly over the through-the-thickness direction is carried out, the expansion is performed over the Ritz coefficients. The expression of the PVD is finally obtained as:

$\delta \mathbf{u}^{\mathrm{T}} \mathbf{K u}=\delta \mathbf{u}^{\mathrm{T}} \mathbf{L}$

where the vector of unknowns is:

$\mathbf{u}^{\mathrm{T}}=\left\{\begin{array}{lllllll}\mathbf{u}_{x 1} & \mathbf{u}_{y 1} & \mathbf{u}_{z 1} & \ldots & \mathbf{u}_{x M} & \mathbf{u}_{y M} & \mathbf{u}_{z M}\end{array}\right\}$

and the stiffness matrix reads:

$\mathbf{K}=\left[\begin{array}{lll}\mathbf{K}_{u_{x} u_{x}} & \mathbf{K}_{u_{x} u_{y}} & \mathbf{K}_{u_{x} u_{z}} \\ \mathbf{K}_{u_{x} u_{y}}^{\mathrm{T}} & \mathbf{K}_{u_{y} u_{y}} & \mathbf{K}_{u_{y} u_{z}} \\ \mathbf{K}_{u_{x} u_{z}}^{\mathrm{T}} & \mathbf{K}_{u_{y} u_{z}}^{\mathrm{T}} & \mathbf{K}_{u_{z} u_{z}}\end{array}\right]$
The explicit expression of all submatrices composing Eq. (41) and of the external loads vector $\mathbf{L}$ is provided in the Appendix (see Eqs. (68) and (71)).

Due to the arbitrariness of the virtual displacements $\delta \mathbf{u}$, the scalar equation of Eq. (39) leads to the solution of a linear system, whose unknowns are the amplitudes of the kinematic variables. Thanks to the sparsity of the stiffness matrix, the solution of the problem is generally performed with a restricted computational effort. Once the amplitudes $\mathbf{u}$ are available, the displacement field, as well as the strain and the in-plane stress field are directly available. Due to the displacement-based approach, further postprocessing is needed to recover the continuity of the transverse stress components. In particular, the indefinite 3D equilibrium equations are integrated along the thickness direction [1,38-40]:

$$
\begin{aligned}
& \sigma_{x z}^{p, k}\left(z_{p}\right)=\sigma_{x z}^{p, k}\left(z_{\mathrm{bot}}\right)-\int_{z_{\mathrm{bot}}}^{z_{p}}\left(\sigma_{x x, x}^{p, k}+\sigma_{x y, y}^{p, k}\right) \mathrm{d} s \\
& \sigma_{y z}^{p, k}\left(z_{p}\right)=\sigma_{y z}^{p, k}\left(z_{\mathrm{bot}}\right)-\int_{z_{\mathrm{bot}}}^{z_{p}}\left(\sigma_{x y, x}^{p, k}+\sigma_{y y, y}^{p, k}\right) \mathrm{d} s \\
& \sigma_{z z}^{p, k}\left(z_{p}\right)=\sigma_{z z}^{p, k}\left(z_{\mathrm{bot}}\right)-\int_{z_{\mathrm{bot}}}^{z_{p}}\left[\int_{0}^{s}\left(\sigma_{x x, x x}^{p, k}+2 \sigma_{x y, x y}^{p, k}+\sigma_{y y, y y}^{p, k}\right) \mathrm{d} \tau\right] \mathrm{d} s
\end{aligned}
$$

The stress derivatives of the components $\sigma_{x x}, \sigma_{x y}$ and $\sigma_{y y}$ in the integrals of Eq. (42) are determined referring to Hooke's law. The integration starts from the bottom-most ply of the composite stack and accounts for the stress boundary conditions applied at the corresponding surface. A good tradeoff between computational cost and accuracy of results is achieved by integrating Eq. (42) with a 4 th order Newton-Cotes rule.

\section{Results}

With the aim of illustrating the potentialities offered by the SGUF-Ritz approach, four test cases from the literature are taken for benchmarking purposes. The first example deals with a laminated cross-ply plate, while the three remaining examples focus on sandwich configurations.

A sketch of a sandwich panel is provided in Fig. 3. The longitudinal and transverse dimensions are $a$ and $b$, the thickness of the single facesheet is $h_{\mathrm{f}}$, and the total panel thickness is $h$. The same sketch holds for the case of a monolithic or laminated plate, where the relevant dimensions are only $a, b$ and $h$. The boundary conditions are specified, from case to case, assuming that the edges of the panel are numbered in the counterclockwise direction, starting from the edge at $x=0$, and $C$ stands for clamped, $S$ for simplysupported and $\mathrm{F}$ for free.

The sublaminate model is denoted by a sequence of acronyms, corresponding to the theory adopted for each of the sublaminates composing the panel. For instance, the sequence $\mathrm{ED}_{332} / \mathrm{LD}_{110}$

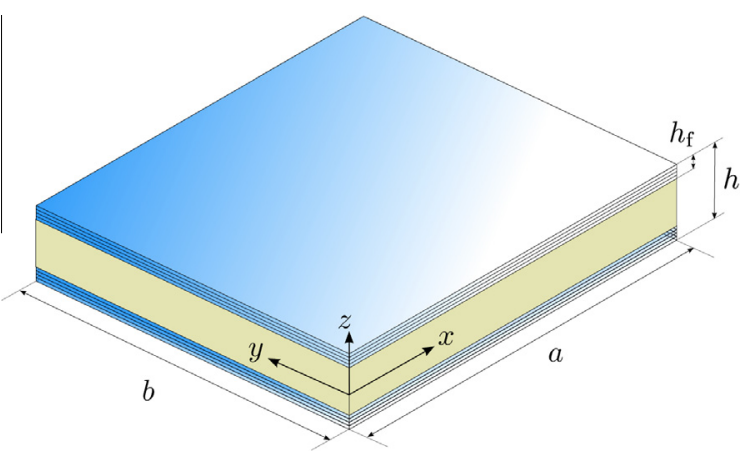

Fig. 3. Sandwich plate. 
indicates that the first sublaminate is modeled according to an Equivalent Single Layer theory $\mathrm{ED}_{332}$, while a layerwise theory $\mathrm{LD}_{110}$ is used for the second sublaminate. The capital ' $\mathrm{D}$ ' in the acronyms indicates that the models are obtained within a displacement-based approach. The three numbers reported as subscripts define the order of the theory for the displacement components $u_{x}, u_{y}$ and $u_{z}$.

The number of plies composing each of the sublaminates cannot be recovered from the acronyms, but will be explicitly described in the text. It is worth emphasizing that throughout this work, the order $N_{u_{z}}^{k}$ used for the expansion of the transverse displacement is taken to be one less than the order $N_{u_{x}}^{k}=N_{u_{y}}^{k}=N_{u_{\alpha}}^{k}(\alpha=x, y)$. This choice allows to obtain a consistent polynomial approximation of the transverse shear strains $\gamma_{\alpha z}^{k}$ of order $N_{u_{\alpha}}^{k}-1=N_{u_{z}}^{k}$. This choice is motivated by the fact that the proposed case studies concern basically the bending response of composite plates, for which transverse shear strains are known to play a relevant role.

The results are checked against reference solutions in terms of displacements and stresses, and different sets of boundary and loading conditions are accounted for. Finally, a set of novel results is proposed for a composite plate under various boundary conditions.

\subsection{Laminated plate with bisinusoidal load}

The first assessment regards the composite plate studied by Vel and Batra [41], where the generalized Eshelby-Stroh formalism is applied to derive 3D solutions. Despite the potentialities of the SGUF formulation can be fully appreciated in the case of sandwich configurations, it is interesting to present a preliminary set of results where the accuracy of the prediction can be demonstrated in detail. In this sense, the benchmarks proposed by Vel and Batra are particularly meaningful, as the 3D results are reported by the authors up to the third/fourth digit, and are available for different set of boundary conditions.
The structure under investigation is a thick square cross-ply plate with a width-to-thickness ratio $a / h$ equal to 5 . The plate is made of a unidirectional composite material with elastic properties $E_{11} / E_{22}=25, G_{12}=G_{12}=0.5 E_{22}, G_{23}=0.2 E_{22}, \quad v_{i k}=0.25$; the stacking sequence is [0/90]. The panel is simply-supported at the two parallel edges at $y=$ const, and is clamped at the two remaining edges. A bisinusoidal load is applied on the top-most ply, i.e., the one oriented at 90, along the positive direction of the axis $z$.

The results are presented in terms of the nondimensional displacement and stress components at the upper and lower surfaces, as well as at the interface between the two laminae. These values are normalized following Ref. [41] as:

$$
\begin{aligned}
& {\left[\bar{u}_{x}(z), \bar{u}_{y}(z)\right]=\frac{100 E_{22} h^{2}}{q a^{3}}\left[u_{x}\left(\frac{a}{4}, \frac{b}{2}, z\right), u_{y}\left(\frac{a}{4}, \frac{b}{2}, z\right)\right]} \\
& \bar{u}_{z}(z)=\frac{100 E_{22} h^{3}}{q a^{4}} u_{z}\left(\frac{a}{2}, \frac{b}{2}, z\right) \\
& {\left[\bar{\sigma}_{x x}(z), \bar{\sigma}_{y y}(z), \bar{\sigma}_{x y}(z)\right]=\frac{10 h^{2}}{q a^{2}}\left[\sigma_{x x}\left(\frac{a}{2}, \frac{b}{2}, z\right), \sigma_{y y}\left(\frac{a}{2}, \frac{b}{2}, z\right), \sigma_{x y}\left(\frac{a}{8}, 0, z\right)\right]} \\
& {\left[\bar{\sigma}_{y z}(z), \bar{\sigma}_{x z}(z)\right]=\frac{10 h}{q a}\left[\sigma_{y z}\left(\frac{a}{2}, 0, z\right), \sigma_{x z}\left(\frac{a}{8}, \frac{b}{2}, z\right)\right]} \\
& \bar{\sigma}_{z z}(z)=\frac{1}{q} \sigma_{z z}\left(\frac{a}{2}, \frac{b}{2}, z\right)
\end{aligned}
$$

The results of the convergence analysis are reported in Table 2, and three distinct layerwise theories, $\mathrm{LD}_{332}, \mathrm{LD}_{554}$ and $\mathrm{LD}_{776}$, are considered. To demonstrate the ability of the proposed approach to obtain highly-accurate results, the number of the shape functions is progressively increased with a step of 5 or 10 terms until convergence is achieved up to the fourth digit for all the quantities. As seen from the table, higher-order theories demand for an

\begin{tabular}{|c|c|c|c|c|c|c|c|c|c|}
\hline Theory & $R$ & $\bar{u}_{x}(h / 2)$ & $\bar{u}_{z}\left(0^{+}\right)$ & $\bar{\sigma}_{x x}(-h / 2)$ & $\bar{\sigma}_{y y}(h / 2)$ & $\bar{\sigma}_{z z}\left(0^{+}\right)$ & $\bar{\sigma}_{x y}(-h / 2)$ & $\bar{\sigma}_{y z}\left(0^{+}\right)$ & $\bar{\sigma}_{x z}\left(0^{+}\right)$ \\
\hline \multicolumn{10}{|l|}{$\mathrm{LD}_{332}$} \\
\hline & 5 & -1.0513 & 1.2117 & -4.5476 & 5.6293 & 0.5736 & 0.3124 & 1.0726 & 1.5283 \\
\hline & 10 & -1.0474 & 1.2154 & -4.6515 & 5.7295 & 0.5790 & 0.3119 & 0.8744 & 1.5514 \\
\hline & 15 & -1.0470 & 1.2155 & -4.6515 & 5.7244 & 0.5797 & 0.3120 & 0.8743 & 1.5560 \\
\hline & 20 & -1.0470 & 1.2155 & -4.6514 & 5.7246 & 0.5790 & 0.3120 & 0.8742 & 1.5539 \\
\hline & 25 & -1.0470 & 1.2155 & -4.6515 & 5.7248 & 0.5791 & 0.3120 & 0.8742 & 1.5541 \\
\hline & 30 & -1.0470 & 1.2155 & -4.6515 & 5.7248 & 0.5790 & 0.3120 & 0.8742 & 1.5540 \\
\hline $3 \mathrm{D}$ & Ref. [41] & -1.0468 & 1.2175 & -4.6300 & 5.7232 & 0.5788 & 0.3132 & 0.8749 & 1.5503 \\
\hline \multicolumn{10}{|l|}{$\mathrm{LD}_{554}$} \\
\hline & 5 & -1.0515 & 1.2125 & -4.4689 & 5.6282 & 0.5733 & 0.3141 & 1.0727 & 1.5287 \\
\hline & 10 & -1.0478 & 1.2169 & -4.6377 & 5.7295 & 0.5779 & 0.3131 & 0.8748 & 1.5517 \\
\hline & 15 & -1.0470 & 1.2171 & -4.6304 & 5.7201 & 0.5790 & 0.3132 & 0.8747 & 1.5527 \\
\hline & 20 & -1.0467 & 1.2171 & -4.6310 & 5.7205 & 0.5789 & 0.3133 & 0.8747 & 1.5519 \\
\hline & 25 & -1.0468 & 1.2171 & -4.6312 & 5.7226 & 0.5794 & 0.3132 & 0.8748 & 1.5521 \\
\hline & 30 & -1.0468 & 1.2171 & -4.6312 & 5.7223 & 0.5788 & 0.3132 & 0.8747 & 1.5518 \\
\hline & 40 & -1.0468 & 1.2171 & -4.6312 & 5.7219 & 0.5788 & 0.3132 & 0.8747 & 1.5520 \\
\hline & 50 & -1.0468 & 1.2171 & -4.6312 & 5.7220 & 0.5788 & 0.3132 & 0.8747 & 1.5520 \\
\hline $3 \mathrm{D}$ & Ref. [41]. & -1.0468 & 1.2175 & -4.6300 & 5.7232 & 0.5788 & 0.3132 & 0.8749 & 1.5503 \\
\hline \multicolumn{10}{|l|}{$\mathrm{LD}_{776}$} \\
\hline & 5 & -1.0515 & 1.2125 & -4.4667 & 5.6286 & 0.5733 & 0.3141 & 1.0727 & 1.5287 \\
\hline & 10 & -1.0478 & 1.2170 & -4.6523 & 5.7306 & 0.5773 & 0.3130 & 0.8748 & 1.5508 \\
\hline & 15 & -1.0472 & 1.2173 & -4.6338 & 5.7197 & 0.5801 & 0.3132 & 0.8748 & 1.5522 \\
\hline & 20 & -1.0467 & 1.2173 & -4.6307 & 5.7199 & 0.5789 & 0.3132 & 0.8748 & 1.5518 \\
\hline & 25 & -1.0468 & 1.2173 & -4.6311 & 5.7239 & 0.5799 & 0.3132 & 0.8748 & 1.5511 \\
\hline & 30 & -1.0468 & 1.2173 & -4.6309 & 5.7236 & 0.5788 & 0.3132 & 0.8748 & 1.5515 \\
\hline & 40 & -1.0468 & 1.2173 & -4.6306 & 5.7222 & 0.5788 & 0.3132 & 0.8748 & 1.5514 \\
\hline & 50 & -1.0468 & 1.2173 & -4.6307 & 5.7227 & 0.5788 & 0.3132 & 0.8748 & 1.5515 \\
\hline & 60 & -1.0468 & 1.2173 & -4.6307 & 5.7226 & 0.5788 & 0.3132 & 0.8748 & 1.5515 \\
\hline $3 D$ & Ref. [41] & -1.0468 & 1.2175 & -4.6300 & 5.7232 & 0.5788 & 0.3132 & 0.8749 & 1.5503 \\
\hline
\end{tabular}
increased number of Ritz functions to reach the desired convergence. Indeed, an expansion of $30 \times 30$ terms is required in the

Table 2

Convergence analysis for a square CSCS plate with $a / h=5$ subjected to bisinusoidal loading applied at the top surface (Ref. [41]) using different theories and $R \times R$ functions. 
Table 3

Comparison with the plate of Ref. [41] using $30 \times 30$ functions and different boundary conditions and plate theories.

\begin{tabular}{|c|c|c|c|c|c|c|c|c|c|c|}
\hline BCs & Theory & $\bar{u}_{x}(h / 2)$ & $\bar{u}_{y}(-h / 2)$ & $\overline{u_{z}}\left(0^{+}\right)$ & $\bar{\sigma}_{x x}(-h / 2)$ & $\bar{\sigma}_{y y}(h / 2)$ & $\bar{\sigma}_{z z}\left(0^{+}\right)$ & $\bar{\sigma}_{x y}(-h / 2)$ & $\bar{\sigma}_{y z}\left(0^{+}\right)$ & $\bar{\sigma}_{x z}\left(0^{+}\right)$ \\
\hline \multicolumn{11}{|c|}{ CSCS } \\
\hline & $\mathrm{ED}_{110}$ & -0.765 & 1.230 & 1.257 & -3.911 & 5.153 & 0.595 & 0.278 & 0.898 & 1.254 \\
\hline & $\mathrm{ED}_{332}$ & -1.026 & 1.306 & 1.182 & -4.345 & 5.551 & 0.587 & 0.300 & 0.839 & 1.452 \\
\hline & $\mathrm{ED}_{776}$ & -1.051 & 1.341 & 1.209 & -4.600 & 5.684 & 0.578 & 0.313 & 0.880 & 1.588 \\
\hline & $\mathrm{LD}_{332}$ & -1.047 & 1.339 & 1.215 & -4.651 & 5.725 & 0.579 & 0.312 & 0.874 & 1.554 \\
\hline & $\mathrm{LD}_{554}$ & -1.047 & 1.341 & 1.217 & -4.631 & 5.722 & 0.579 & 0.313 & 0.875 & 1.552 \\
\hline & $\mathrm{LD}_{776}$ & -1.047 & 1.341 & 1.217 & -4.631 & 5.724 & 0.579 & 0.313 & 0.875 & 1.552 \\
\hline & 3D Ref. [41] & -1.047 & 1.341 & 1.217 & -4.630 & 5.723 & 0.579 & 0.313 & 0.875 & 1.550 \\
\hline \multicolumn{11}{|c|}{ SSSS } \\
\hline & $\mathrm{ED}_{110}$ & -1.737 & 1.737 & 1.758 & -7.157 & 7.157 & 0.500 & 0.485 & 1.220 & 1.128 \\
\hline & $\mathrm{ED}_{332}$ & -1.845 & 1.869 & 1.680 & -7.510 & 7.721 & 0.495 & 0.518 & 1.175 & 1.188 \\
\hline & $\mathrm{ED}_{776}$ & -1.875 & 1.902 & 1.703 & -7.634 & 7.856 & 0.495 & 0.527 & 1.219 & 1.225 \\
\hline & $\mathrm{LD}_{332}$ & -1.870 & 1.898 & 1.712 & -7.681 & 7.905 & 0.495 & 0.527 & 1.211 & 1.216 \\
\hline & $\mathrm{LD}_{554}$ & -1.870 & 1.899 & 1.712 & -7.671 & 7.894 & 0.495 & 0.527 & 1.211 & 1.216 \\
\hline & $\mathrm{LD}_{776}$ & -1.870 & 1.899 & 1.712 & -7.671 & 7.894 & 0.495 & 0.527 & 1.211 & 1.216 \\
\hline & 3D Ref. [41] & -1.870 & 1.899 & 1.712 & -7.671 & 7.894 & 0.495 & 0.527 & 1.211 & 1.216 \\
\hline \multicolumn{11}{|c|}{ FSFS } \\
\hline & $\mathrm{ED}_{110}$ & -0.538 & 2.899 & 2.777 & -2.469 & 11.907 & 0.363 & 0.111 & 1.487 & 0.334 \\
\hline & $\mathrm{ED}_{332}$ & -0.558 & 3.202 & 2.690 & -2.570 & 12.501 & 0.356 & 0.108 & 1.475 & 0.429 \\
\hline & $\mathrm{ED}_{776}$ & -0.566 & 3.297 & 2.743 & -2.642 & 12.837 & 0.358 & 0.108 & 1.549 & 0.424 \\
\hline & $\mathrm{LD}_{332}$ & -0.564 & 3.291 & 2.753 & -2.666 & 12.889 & 0.358 & 0.108 & 1.541 & 0.416 \\
\hline & $\mathrm{LD}_{445}$ & -0.565 & 3.291 & 2.753 & -2.661 & 12.878 & 0.358 & 0.108 & 1.541 & 0.415 \\
\hline & $\mathrm{LD}_{776}$ & -0.565 & 3.291 & 2.753 & -2.661 & 12.877 & 0.358 & 0.108 & 1.541 & 0.415 \\
\hline & 3D Ref. [41] & -0.565 & 3.291 & 2.753 & -2.660 & 12.877 & 0.359 & 0.108 & 1.541 & 0.416 \\
\hline
\end{tabular}

case of the $\mathrm{LD}_{332}$ theory, while $60 \times 60$ are needed when considering the $\mathrm{LD}_{776}$ theory. Thanks to the efficient implementation of the method, the S-GUF-Ritz can be easily adopted to perform highfidelity analyses, where high-order theories are used in conjunction with several Ritz functions. For practical purposes, the convergence can be established by checking the percent change with respect the results obtained in the previous iterations. In this case, $15 \times 15$ functions would suffice to obtain a maximum difference of $0.5 \%$ with respect to the results obtained with $R=10$.

The accuracy of the S-GUF-Ritz results can be noticed from the comparison with the 3D results obtained by Vel and Batra. A very close agreement is observed for the three considered LW theories, i.e., $\mathrm{LD}_{332}, \mathrm{LD}_{554}$ and $\mathrm{LD}_{776}$, with maximum percent differences equal to $0.50 \%, 0.11 \%$ and $0.07 \%$, respectively.

Additional results are presented in Table 3 to verify the ability of the S-GUF-Ritz method to properly handle different boundary conditions and to illustrate their effect on the plate response. The geometric and elastic properties of the plate, as well as the loading condition, are unchanged with respect to the previous set of results. In this case, the number of shape functions is taken equal to $30 \times 30$, a number of terms sufficient to guarantee a percent difference below $0.2 \%$ with respect to the solution obtained using $R=S=25$. The results are now reported by considering both ESL and LW theories. A shear correction of $5 / 6$ is used for the $\mathrm{ED}_{110}$ theory (FSDT), while it is set equal to one in the remaining cases.

Overall, it is clear that the adoption of the $\mathrm{ED}_{110}$ theory determines a significant discrepancy with $3 \mathrm{D}$ predictions, and even the adoption of $\mathrm{ED}_{332}$ leads to percent differences as high as $6 \%$. The quality of the prediction is improved when $\mathrm{ED}_{776}$ is used, but it can be observed that the results relative to the normal stress $\bar{\sigma}_{z z}$ are still $2 \%$ higher than 3D predictions. On the other hand, layerwise results are extremely accurate even for the lowest order here considered, i.e. $\mathrm{LD}_{332}$. The improvement associated with the adoption of theories $\mathrm{LD}_{445}$ and $\mathrm{LD}_{776}$ is restricted to the third digits. As already highlighted for the results of Table 2, the predictions obtained using $\mathrm{LD}_{776}$ are almost identical to 3D results: all but six of the results in Table 3 are identical to the 3D solution of Ref. [41] up to the third digit.

The through-the-thickness distribution of the transverse shear stresses $\sigma_{x z}$ and $\sigma_{y z}$ is reported in Fig. 4, where the comparison is presented against the results of Ref. [41]. The curves of Fig. 4 are relative to the in-plane position $x=a / 20, y=b / 2$, while the curves of Fig. 4 describe the plate response at the position $x=a / 20, y=0$. The results are computed by using $30 \times 30$ functions and LD $_{776}$ theory. Boundary conditions of CSCS and FSFS are assessed in Fig. 4, while CSCS and SSSS conditions are considered in Fig. 4. The S-GUF-Ritz results are again in excellent agreement with the 3D predictions reported by Vel and Batra. As seen from Fig. 4, the transverse stress distribution, for all the boundary conditions here considered, is not parabolic, but is characterized by a discontinuity of the first derivative at the interface between the first and the second ply. It can be observed that, depending on the specific boundary condition, the shape of the stress distribution changes accordingly. For instance, a stress reversal effect, which is correctly captured by the present approach, can be observed at the bottom of the CSCS ply in Fig. 4.

\subsection{Sandwich plates with bisinusoidal load}

The second set of test cases regards the sandwich panels studied by Cho and Averill [5], denoted in the next as S1 and S2 panels. In both cases the structure is a composite sandwich construction subjected to a bisinusoidally distributed pressure of magnitude $q$ at the top surface, and simply supported at the four edges. The panel S1 is a standard sandwich configuration with a 5-ply facesheet and a relatively weak core, while the panel S2 is a complex multilayered panel with 55 plies made of 5 different materials.

\subsubsection{Panel S1}

Panel S1 is a thick square plate, characterized by a ratio $a / h$ equal to 4 , and $h_{\mathrm{f}} / h$ equal to 0.1 . The elastic properties of the materials are summarized in Table 4, where M1 to M3 identify the materials of the facesheets, and $\mathrm{C} 1$ is the material used for the core. The stacking sequence, in terms of materials used for the eleven plies and their relative thickness, is presented in Table 5. Note that the material properties are reported in the laminate reference system, thus all the plies are assumed to be oriented at 0 .

The S-GUF-Ritz results are computed by using two models with a similar number of Theory-related Degrees Of Freedom (T-DOF). Goal of this assessment is to highlight the advantages related to 


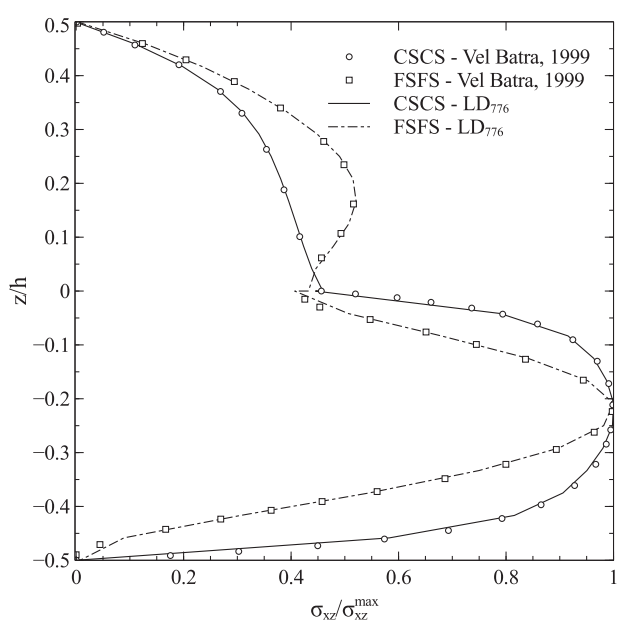

(a)

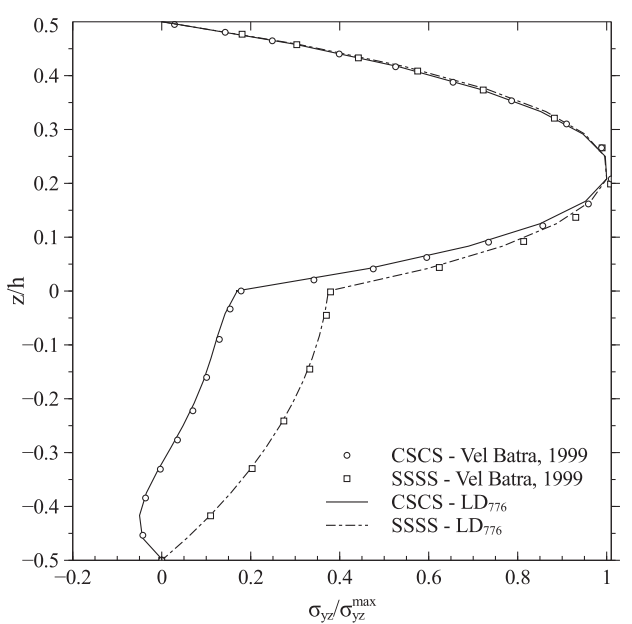

(b)

Fig. 4. Transverse shear stresses versus nondimensional thickness coordinate for square [0/90] laminate with different boundary conditions: (a) $\sigma_{x z} / \sigma_{x z}^{\max }$ at $x=a / 20, y=b / 2$, (b) $\sigma_{y z} / \sigma_{y z}^{\max }$ at $x=a / 20, y=0$.

Table 4

Material elastic properties for sandwich panel S1 (Ref. [5]).

\begin{tabular}{lllll}
\hline & M1 & M2 & M3 & C1 \\
\hline $\mathrm{E}_{11}(\mathrm{MPa})$ & 6900 & 224800 & 172400 & 345 \\
$\mathrm{E}_{22}(\mathrm{MPa})$ & 172400 & 69000 & 69000 & 1034 \\
$\mathrm{E}_{33}(\mathrm{MPa})$ & 6900 & 69000 & 69000 & 345 \\
$\mathrm{G}_{12}(\mathrm{MPa})$ & 3450 & 56600 & 3450 & 150 \\
$\mathrm{G}_{13}(\mathrm{MPa})$ & 1380 & 56000 & 3450 & 150 \\
$\mathrm{G}_{23}(\mathrm{MPa})$ & 3450 & 3450 & 1380 & 290 \\
$v_{12}$ & 0.01 & 0.10 & 0.25 & 0.01 \\
$v_{13}$ & 0.25 & 0.10 & 0.25 & 0.15 \\
$v_{23}$ & 0.25 & 0.25 & 0.25 & 0.15 \\
\hline
\end{tabular}

the subdivision of the panel into sublaminates, and the improved accuracy of the results in comparison to an equivalent single theory with a similar number of degrees of freedom.
The first model to be considered is FSDT/ED ${ }_{332} /$ FSDT and is based upon the definition of three sublaminates: the first-order shear deformation theory is assumed for the two facesheets, while the core is modeled according to an $\mathrm{ED}_{332}$ theory. The second model employs one single sublaminate for which the high-order ESL theory $\mathrm{ED}_{554}$ is adopted. Note that the two approaches have a comparable number of T-DOF, 15 and 17, respectively, thus yielding a similar total size of the problem. In both cases, the results were found to be converged with a number of $50 \times 50$ shape functions.

The through-the-thickness distribution of the in-plane stress components $\sigma_{x x}$ and $\sigma_{x y}$ is plotted in Fig. 5, where the 3D elasticity solution taken from Ref. [5] is reported. For clarity purposes, the region relative to the facesheet is shaded in light gray. A very good matching can be appreciated between the elasticity solution and

Table 5

Lay-up of sandwich panel S1 of Ref. [5].

\begin{tabular}{|c|c|c|c|c|c|c|c|c|c|c|c|}
\hline$h_{p} / h$ & 0.01 & 0.025 & 0.015 & 0.020 & 0.030 & 0.800 & 0.03 & 0.02 & 0.015 & 0.025 & 0.01 \\
\hline Material & M1 & M2 & M3 & M1 & M3 & $\mathrm{C} 1$ & M3 & M1 & M3 & M2 & M1 \\
\hline
\end{tabular}

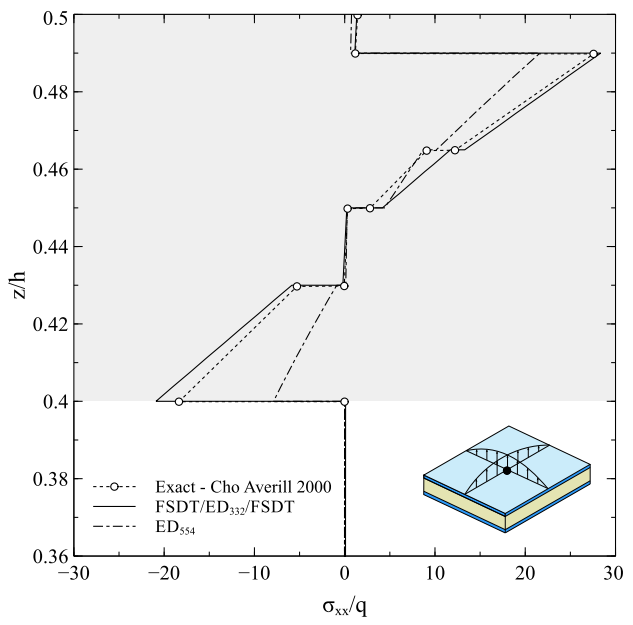

(a)

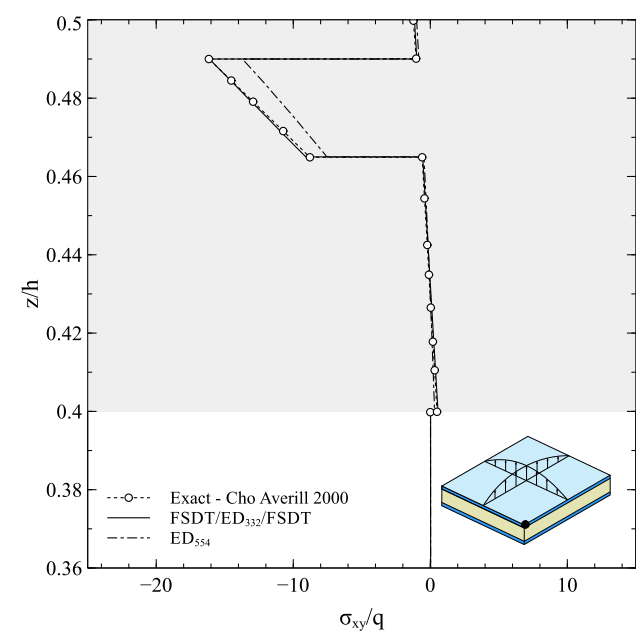

(b)

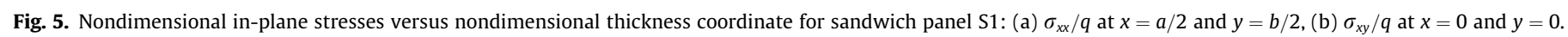




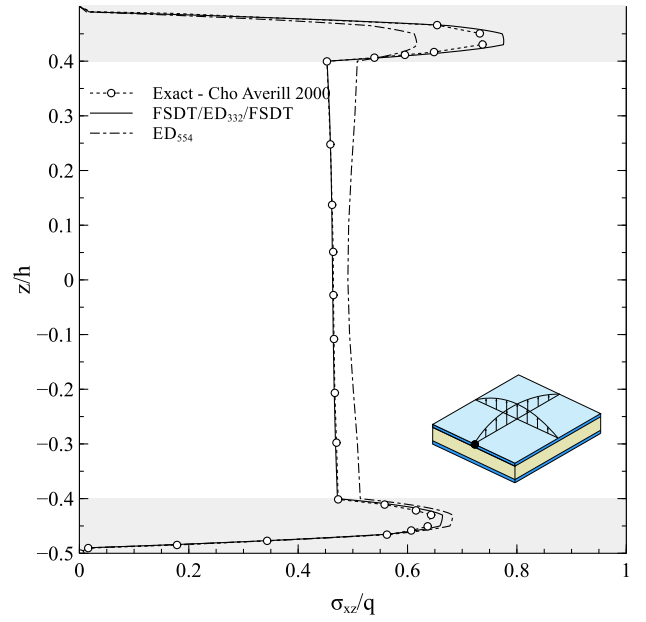

(a)

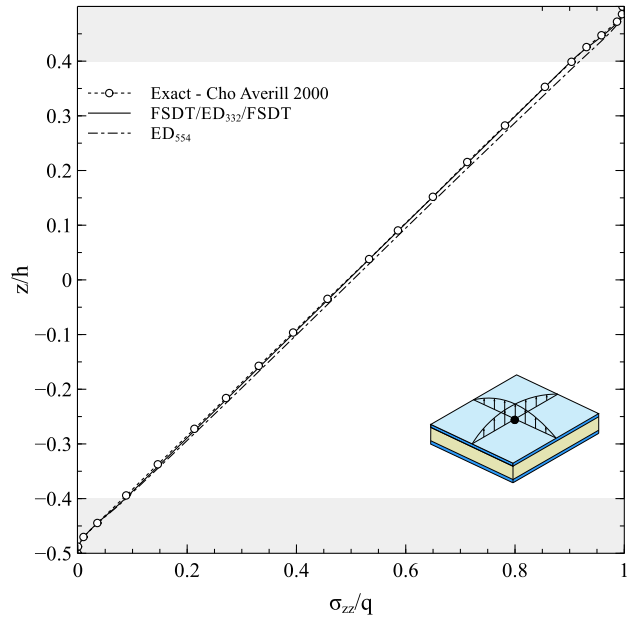

(b)

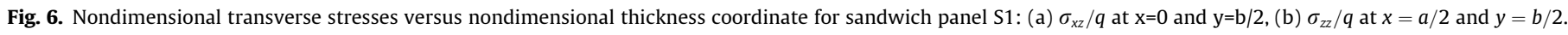

the FSDT/ED ${ }_{332} /$ FSDT, for both the direct and the shear stress components $\sigma_{x x}$ and $\sigma_{x y}$. On the contrary, the results computed with the pure ESL approach display significant deviations from the reference solution and an underestimation of the minimum stress $\sigma_{x x}$ as high as a $50 \%$, see Fig. 5. Fig. 6 presents the plot of the transverse stress components $\sigma_{x z}$ and $\sigma_{z z}$. Close matching can be observed for both stress components between the exact solution and the distributions obtained with the FSDT/ED ${ }_{332} /$ FSDT model. On the contrary, the $\mathrm{ED}_{554}$ model overestimates $\sigma_{x z}$ in the lower facesheet and the core and underestimates it in the upper facesheet. The advantage of adopting a sublaminate description is thus clear.

\subsubsection{Panel S2}

The configuration of Panel S2 is taken from the work of Cho and Averill [5] and is inspired from the panels of the US Army Composite Armored Vehicle (CAV). The panel is square and has a width-to-thickness ratio equal to 4 ( $a=b=172 \mathrm{~mm}$, total thickness $h=43 \mathrm{~mm}$ ). The laminate is obtained by stacking 55 plies made of five different materials, whose elastic properties are summarized in Table 6 . The elastic properties of the three glass fiber materials are quite similar. On the other hand, the ceramic has good in-plane properties but relatively weak transverse moduli, while the rubber is characterized by weak mechanical properties in all the directions. Based on this observation, the panel can be subdivided into three distinct regions: the inner shell, consisting in the first 41 plies, the armor core, composed of the rubber and ceramic layer, and the outer shell, composed of the remaining 12 plies.

A sketch of the panel is provided in Fig. 7. The figure illustrates the distribution of the materials along the thickness direction, the

\section{Table 6}

Material elastic properties for the sandwich panel S2 (composite armored vehicle CAV) of Ref. [5].

\begin{tabular}{llllll}
\hline & $\begin{array}{l}\text { Glass fabric } \\
\text { A (GFA) }\end{array}$ & $\begin{array}{l}\text { Glass } \\
\text { tow }(\mathrm{GT})\end{array}$ & $\begin{array}{l}\text { Glass fabric } \\
\text { B (GFB) }\end{array}$ & Rubber & Ceramic \\
\hline $\mathrm{E}_{11}(\mathrm{MPa})$ & 20700 & 42700 & 20700 & 21 & 34500 \\
$\mathrm{E}_{22}(\mathrm{MPa})$ & 20700 & 6900 & 20700 & 21 & 34500 \\
$\mathrm{E}_{33}(\mathrm{MPa})$ & 8270 & 6900 & 7580 & 21 & 860 \\
$\mathrm{G}_{12}(\mathrm{MPa})$ & 6900 & 2070 & 6900 & 6.90 & 17240 \\
$\mathrm{G}_{13}(\mathrm{MPa})$ & 3170 & 2070 & 2690 & 6.90 & 59 \\
$\mathrm{G}_{23}(\mathrm{MPa})$ & 3170 & 2070 & 2690 & 6.90 & 59 \\
$v_{12}$ & 0.13 & 0.29 & 0.13 & 0.45 & 0.15 \\
$v_{13}$ & 0.18 & 0.29 & 0.18 & 0.45 & 0.15 \\
$v_{23}$ & 0.18 & 0.37 & 0.18 & 0.45 & 0.15 \\
\hline
\end{tabular}

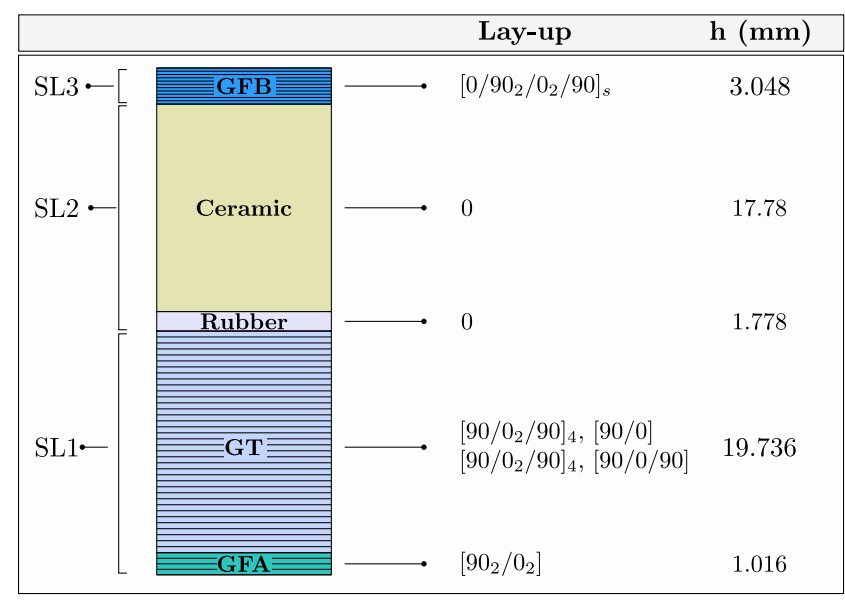

Fig. 7. Stacking sequence and sublaminate representation of the sandwich panel S2.

angles of orientations, and the thicknesses of the various regions. Furthermore, the figure reports the idealization of the structure into three sublaminates, which is successively used for the analysis: the first and the third sublaminates correspond to the inner and the outer shells, respectively, while the second sublaminate is representative of the core region.

Two models are considered for the analysis: $\mathrm{ED}_{554}$ is a high order ESL theory, while FSDT/LD $\mathrm{L}_{332} /$ FSDT is a typical S-GUF model, through which a simple FSDT kinematics is used for the outer and inner shells and a high order LW model is adopted for the armor core. The choice of a layerwise description for the core is motivated by the large difference between the elastic properties of the ceramic and rubber plies constituting the armor. The number of T-DOF is 17 for the former model and 23 for the latter one. The number of shape functions is $50 \times 50$.

The in-plane stresses are reported in Fig. 8, where the light gray area is relative to the glass-fiber facesheets, while the diagonal cross pattern highlights the thin rubber layer region. The results are compared to the exact solution reported by Cho and Averill (note that all the plies are oriented at 0 and 90, thus an exact solution of the 3D problem can be derived). As seen from the plot of $\sigma_{x x}$ in Fig. 8, the $\mathrm{ED}_{554}$ model, despite the relatively high-order of the expansion, cannot properly capture the trend. Even the maximum and the minimum stresses, which can be interesting parameters 


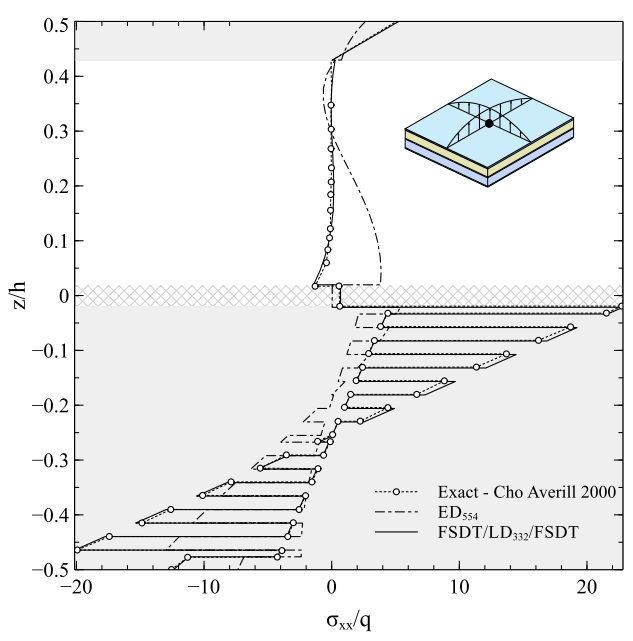

(a)

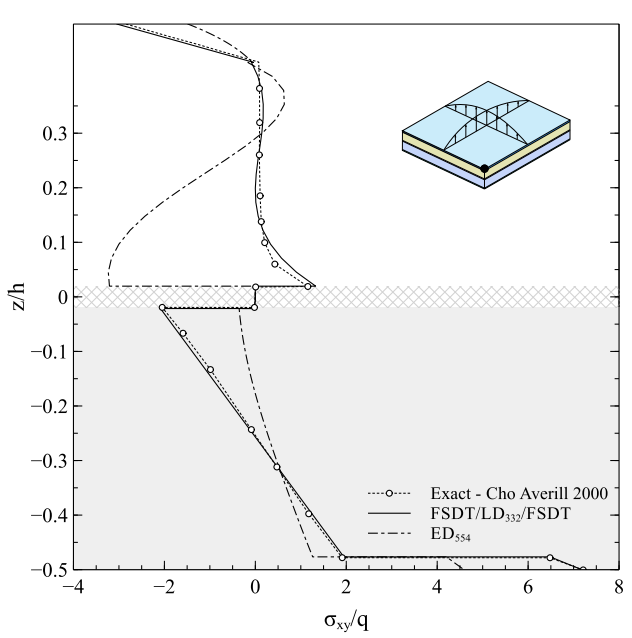

(b)

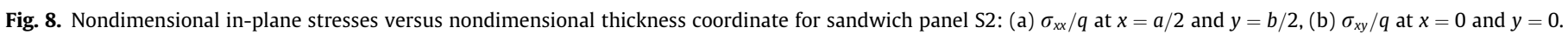

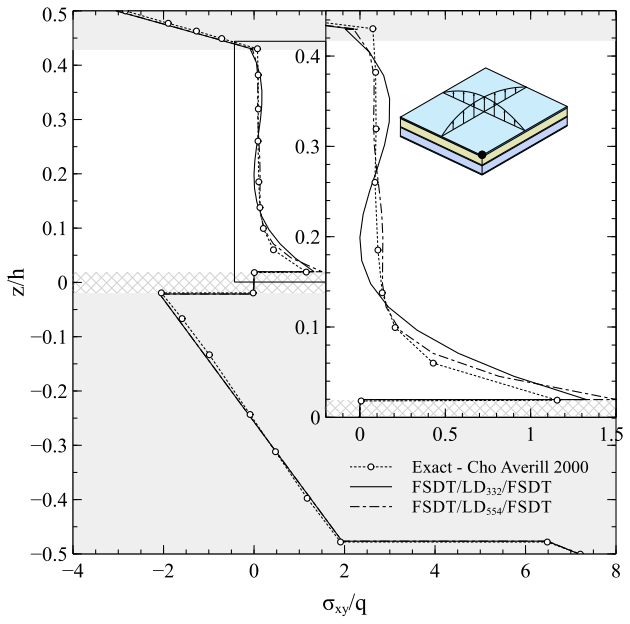

(a)

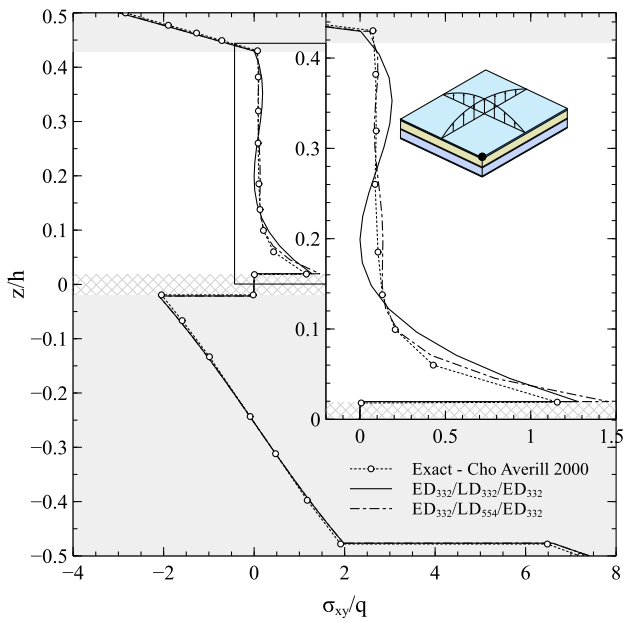

(b)

Fig. 9. Comparison between different theories for shear stress $\sigma_{x y} / q$ at $x=0$ and $y=0$ (sandwich panel S2).

for design purposes, are poorly predicted. On the other hand, the proposed sublaminate description FSDT/LD $332 /$ FSDT allows for a refined stress analysis, and the results are almost identical to the exact ones. The same considerations hold for the shear stress at the plate corner, whose plot is presented in Fig. 8. In this case, a slight discrepancy between FSDT/LD $\mathrm{L}_{332} /$ FSDT and exact solution can be observed in the armor core region. While the quality of these predictions is still retained satisfactory, it is interesting to investigate the possibility of achieving even more detailed predictions by improving the order of the plate theories of the S-GUF model. More specifically, the effect increasing the order of the core is made clear in Fig. 9. In this case, additional results are reported referring to the model FSDT/LD ${ }_{554} /$ FSDT. As seen, they are in close agreement with the exact solution, and the oscillating behavior observed with the FSDT/LD L $_{32} /$ FSDT model is substantially reduced. In the topmost part of the zoom area, a small difference can be observed between the refined and the exact solution, due to the inability of the FSDT/LD ${ }_{554} /$ FSDT model to capture the abrupt change of slope between the core and the upper facesheet. This minor discrepancy can be removed, as highlighted in Fig. 9, by increasing the order of the theory in the facesheet region. In fact, the results obtained with the $\mathrm{ED}_{332} / \mathrm{LD}_{554} / \mathrm{ED}_{332}$ theory are almost indistinguishable from the exact ones. This latter model is characterized by a relatively high number of T-DOF, that is equal to 47 , but, at same time, the accuracy of the predictions is comparable to a more expensive 3D solution. Indeed one of the advantages of the proposed S-GUF-Ritz method is the possibility of deriving quasi-3D predictions even in the case of stacking sequence or boundary conditions for which 3D exact solutions cannot be derived.

The response in terms of transverse stresses is presented in Fig. 10, where both the shear and the normal components $\sigma_{x z}$ and $\sigma_{z z}$ are reported. Despite the recovery of the transverse stresses is performed by integration of the 3D equations - which has the effect of improving the quality of the prediciton even in the case of relatively low-order models - the quality of the results obtained with the $\mathrm{ED}_{554}$ model is rather poor. On the contrary, the adoption of the sublaminate description FSDT/LD ${ }_{332} /$ FSDT provides high-quality results, in good agreement with the 3D exact solution. For completeness, the results are presented also in terms of normal deformation $\varepsilon_{z z}$ in Fig. 11. In this case, the facesheets cannot be modeled using FSDT, as far as the zero order description of the transverse displacement is associated with a null transverse deformation. For this reason, the results are reported with a 


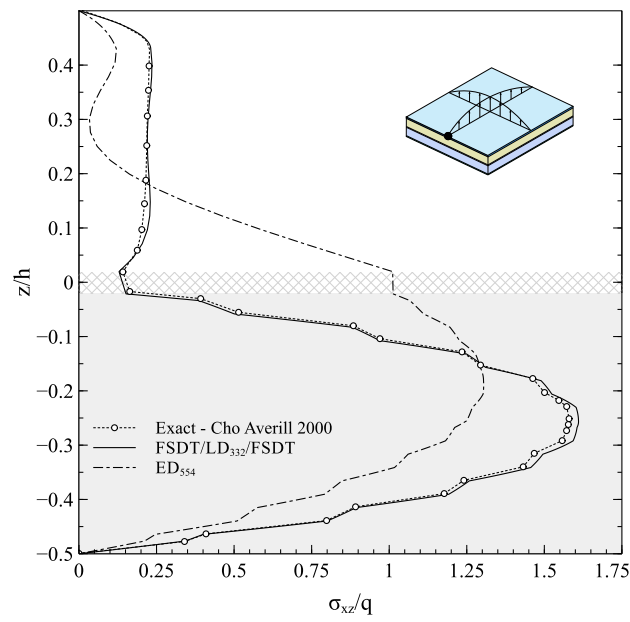

(a)

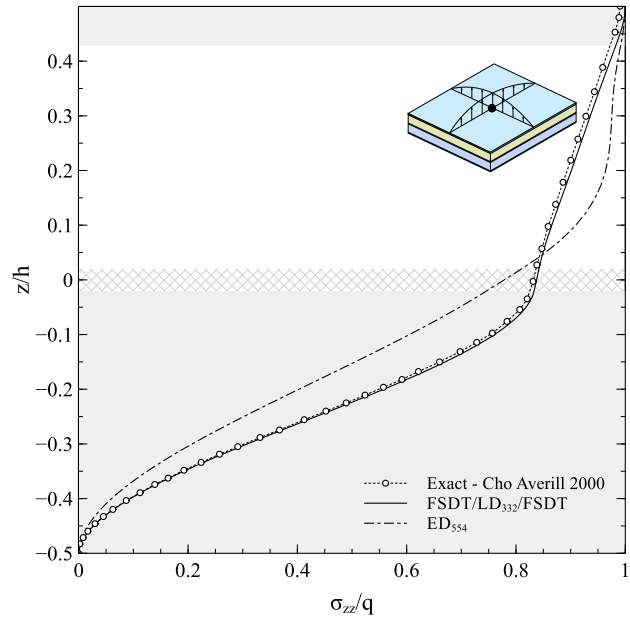

(b)

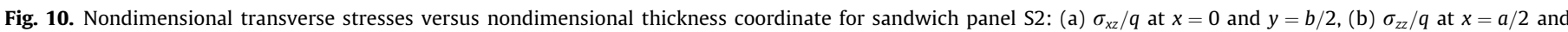
$y=b / 2$.

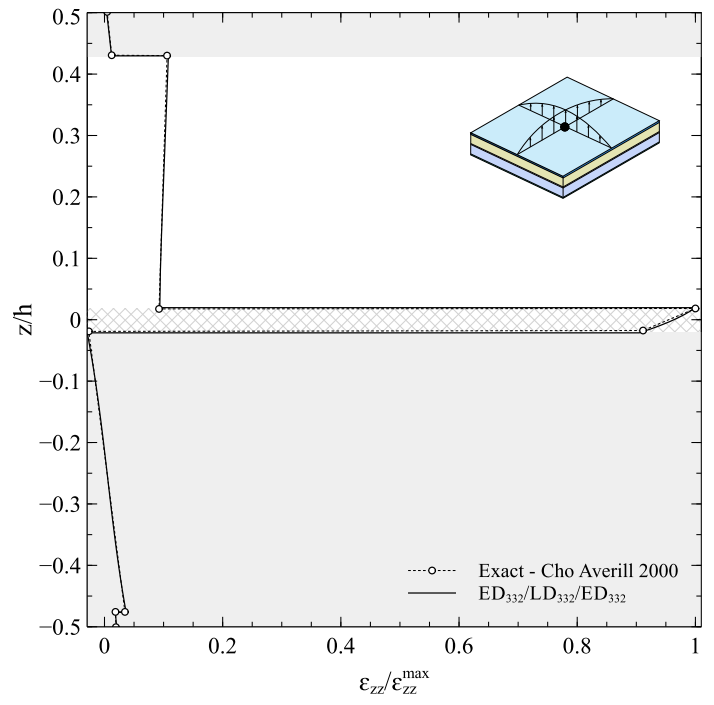

Fig. 11. Transverse normal strain $\varepsilon_{z z} / \varepsilon_{z z \text { max }}$ versus nondimensional thickness coordinate at $x=a / 2$ and $y=b / 2$ (sandwich panel S2).

sublaminate model $\mathrm{ED}_{332} / \mathrm{LD}_{332} / \mathrm{ED}_{332}$. As expected, the highest values of $\varepsilon_{z z}$ are experienced by the panel in correspondence of the rubber layer, i.e. the material with the weakest elastic properties. Noticeable transverse deformation is observed also in the ceramic part of the armor core, where the transverse elastic modulus is approximately one order of magnitude smaller in comparison to the glass fiber plies of the outer facesheets. Even in this case, the results are very close to the reference solution, demonstrating the quality of the results obtained with the S-GUF-Ritz method.

\subsection{Sandwich plate with localized load}

\subsubsection{Panel S3}

The challenging test case proposed by Barut et al. [39] is next investigated, which permits to further highlight the capabilities offered by the S-GUF-Ritz method. In this case, the sources of complexity are, on the one hand the abrupt change of mechanical properties along the thickness direction, on the other hand the presence
Table 7

Material elastic properties for sandwich panel S3 (Ref. [39]).

\begin{tabular}{llll}
\hline & Carbon/Epoxy & Honeycomb & PVC foam \\
\hline $\mathrm{E}_{11}(\mathrm{MPa})$ & 157890 & 0.3067 & 103 \\
$\mathrm{E}_{22}(\mathrm{MPa})$ & 9584 & 0.2779 & 103 \\
$\mathrm{E}_{33}(\mathrm{MPa})$ & 9584 & 3833 & 103 \\
$\mathrm{G}_{12}(\mathrm{MPa})$ & 5957 & 23 & 40 \\
$\mathrm{G}_{13}(\mathrm{MPa})$ & 5957 & 757 & 40 \\
$\mathrm{G}_{23}(\mathrm{MPa})$ & 2537 & 633 & 40 \\
$v_{12}$ & 0.32 & 0.99 & 0.30 \\
$v_{13}$ & 0.32 & $3 \times 10^{-5}$ & 0.30 \\
$v_{23}$ & 0.48 & $3 \times 10^{-5}$ & 0.30 \\
\hline
\end{tabular}

of a localized loading condition. This latter aspect can be particularly difficult to be handled in the context of a formulation based on the use of global shape functions. However, as demonstrated next, the efficiency of the implementation allows to easily consider a high number of functions, thus making it possible to capture even local effects.

The panel under investigation is square and characterized by a width-to-thickness ratio of 10 . Simply-supported boundary conditions are considered at the four edges, while the load is a localized pressure of intensity $q=-1000 \mathrm{lb} / \mathrm{in}^{2}$, applied at the top surface of the panel and acting opposed to the positive direction of the $z$-axis. The loaded area is a square of dimension $a / 10 \times a / 10$ located at the plate center. The thickness of each facesheet is equal to $10 \%$ of the total panel thickness, i.e. $h_{\mathrm{f}} / h=0.1$, thus the core corresponds to $80 \%$ of the total thickness. The facesheets are cross-ply laminates made of carbon/epoxy plies stacked as $[0 / 90]_{5}$ (outer plies oriented at 0 ). Two distinct materials are considered for the core, namely a soft PVC foam and a honeycomb. The elastic properties of all materials are taken from [39] and summarized in Table 7.

The results are computed with a number of $60 \times 60$ shape functions, chosen on the basis of a preliminary convergence analysis. Two kinematic formulations are considered for this example, $\mathrm{ED}_{554}$ and FSDT/ED $332 / F S D T$, where in the latter model the sublaminates represent the two facesheets and the core.

All results are compared against the exact 3D results reported by Barut et al. [39] which, in turn, are obtained by implementing the exact solution of Anderson et al. [42]. The deformed configuration is illustrated in Fig. 12 with regard to the panel cross-section at $y=b / 2$ for the two core materials. It can be seen that the displacement of the honeycomb-core panel is smaller than that of 


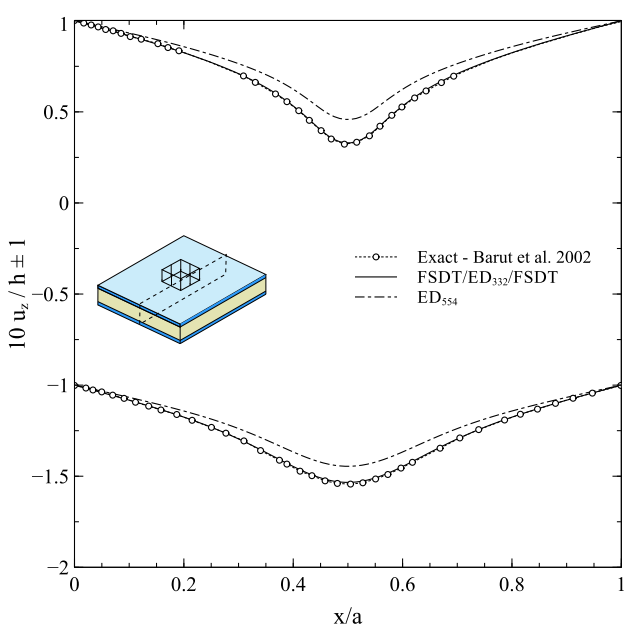

(a)

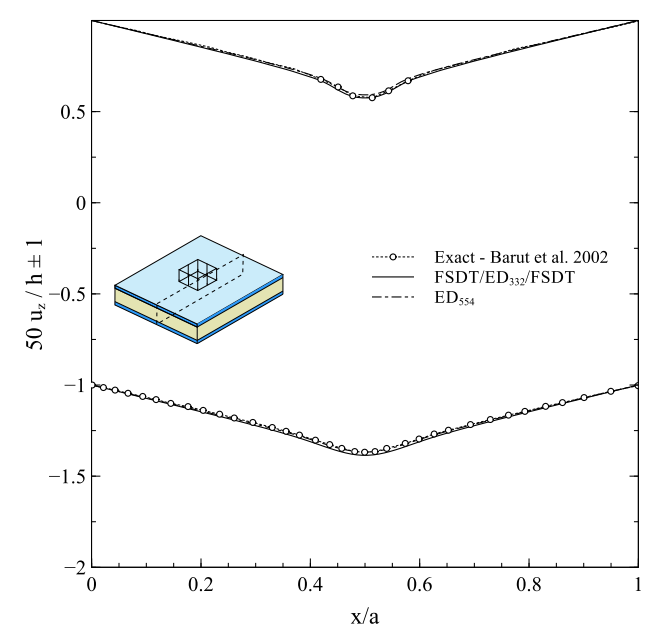

(b)

Fig. 12. Transverse displacement of upper and lower facesheets at $y=b / 2$ for sandwich panel S3 with: (a) PVC core, (b) honeycomb core.

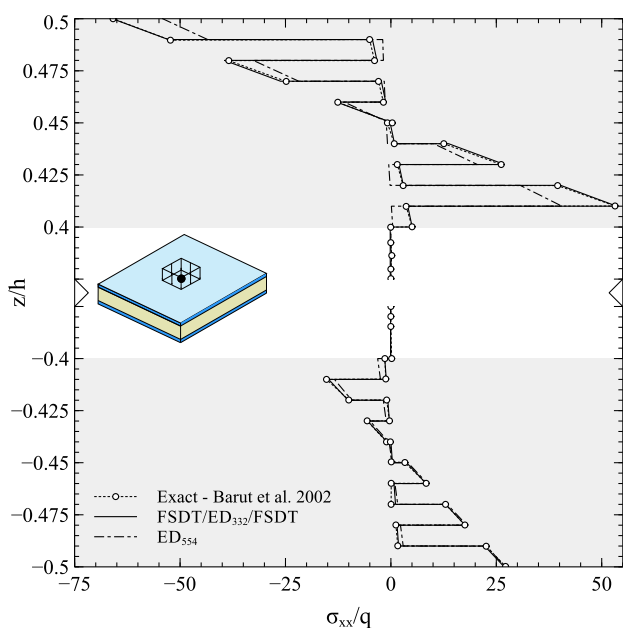

(a)

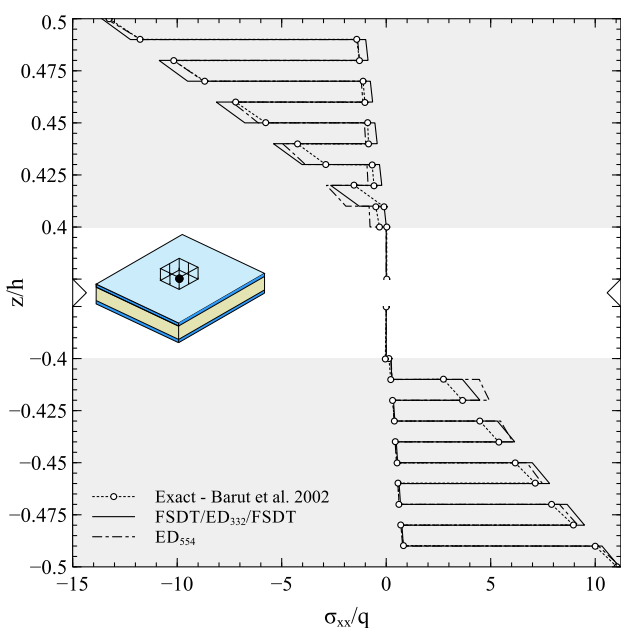

(b)

Fig. 13. Normal stresses $\sigma_{x x} / q$ versus nondimensional thickness coordinate at $x=a / 2, y=b / 2$ for sandwich panel S3 with: (a) PVC core, (b) honeycomb core.

the panel with the PVC core. The local indentation due to the local load introduction is clearly visible in both cases, with a more pronounced effect on the top facesheets. The results of the FSDT/ $\mathrm{ED}_{332} / \mathrm{FSDT}$ model are in close agreement with the exact solution, except from a negligibly small overestimation of the transverse displacement of the bottom facesheet of the honeycomb core panel. The results obtained with the $\mathrm{ED}_{554}$ model are very close to exact solution in the case of the honeycomb core, but the predictions are not accurate for the PVC-core configuration. As seen from Fig. 12, the underestimation of the transverse displacement at the upper and lower facesheets is noticeable.

The through-the-thickness behavior of $\sigma_{x x}$ at the center of the panel is reported in Fig. 13. The plots are restricted to the facesheet region, and the comparison is illustrated against the 3D exact solution. Overall, the quality of the FSDT/ED ${ }_{332} /$ FSDT results is higher in comparison to the $\mathrm{ED}_{554}$ model. In particular, the comparison with the exact solution reveals nearly coincident results for the panel with the PVC core, while close agreement - although not identical results - can be seen in the case of the honeycomb core. It is interesting to observe a significantly different mechanical response between the panel with honeycomb core and that with PVC core. In the former case, the top and bottom facesheets are entirely subjected to a compressive and tensile stress state, respectively, see Fig. 13. This behavior is significantly different from what is observed in Fig. 13 for the case of the PVC core: here, the core is responsible for a poor load transfer between the facesheets, that are thus subjected to a localized bending as testified by the coexistence of tensile and compressive stresses in both the facesheets.

The transverse stresses $\sigma_{x z}$, evaluated at $x=\mathrm{a} / 2$ and $y=\mathrm{b}$, are presented in Fig. 14. In this case, the two theories lead to similar predictions, which are in substantial agreement with the reference solution. On the contrary, some differences between the two theories can be seen in terms of the transverse normal stress, as reported in Fig. 15. The discrepancy between FSDT/ED H32 $_{32} / \mathrm{FSDT}$ and $\mathrm{ED}_{554}$ results is more evident for the PVC core. In this case, the weakest transverse elastic moduli is responsible for noticeable kinks at the face/core interface. While these sudden changes of slope are properly predicted by the three-sublaminate model, the ESL model tends to smooth the effect and provides less accurate 


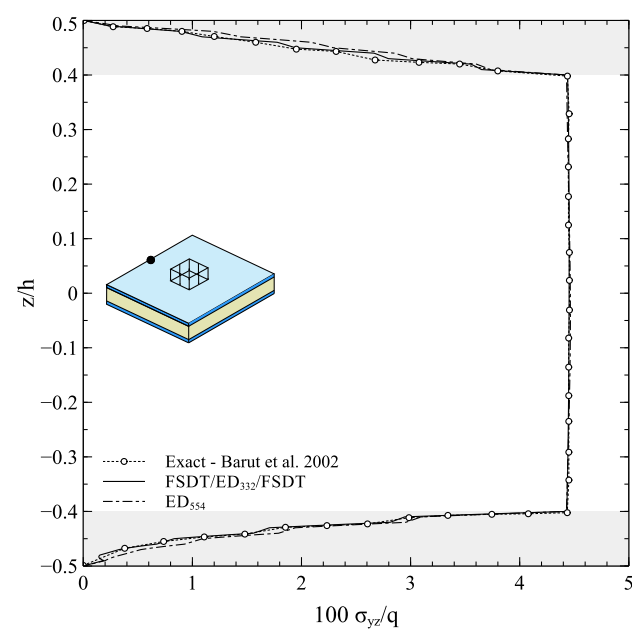

(a)

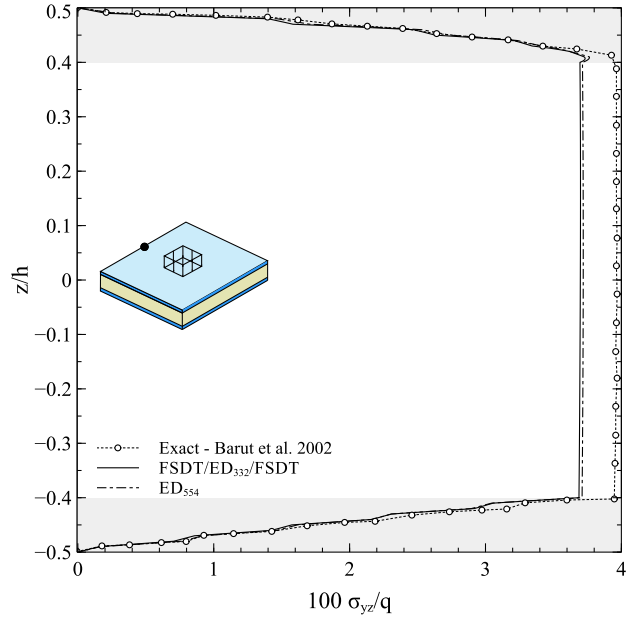

(b)

Fig. 14. Transverse stresses $\sigma_{y z} / q$ versus nondimensional thickness coordinate at $x=a / 2, y=b$ for sandwich panel S3 with: (a) PVC core, (b) honeycomb core.

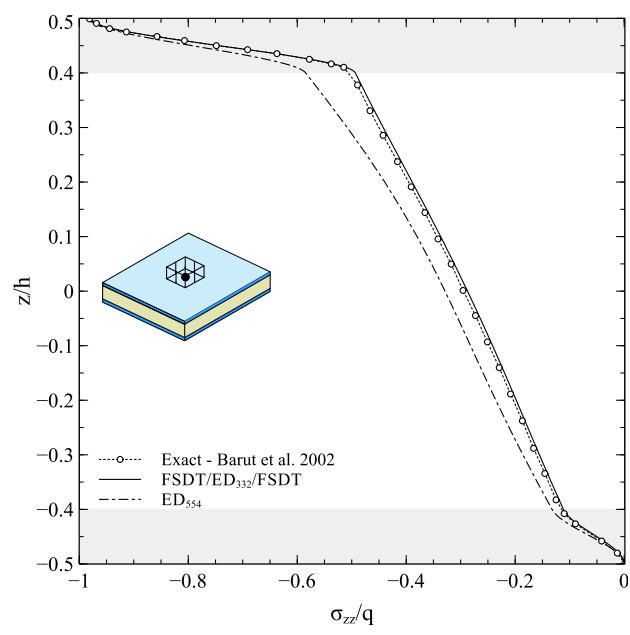

(a)

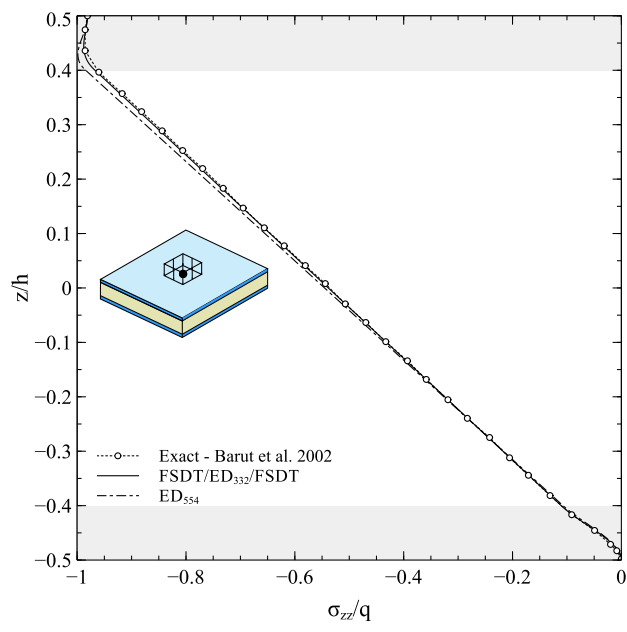

(b)

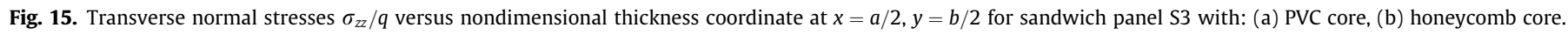

results. This behavior is less evident for the honeycomb-core panel, where the change of elastic properties along the thickness direction is milder. It follows that the normal stress is characterized by a smoother response, which can be correctly predicted even in the context of a single sublaminate model.

\subsection{Additional benchmark results}

In the previous sections, close agreement between S-GUF-Ritz and 3D results has been demonstrated in those cases for which the elasticity problem can be solved in exact manner. In order to exploit the potentialities of the formulation here proposed, a set of novel results is now presented for a panel, here denoted as S4, subjected to different sets of boundary and loading conditions. These results should prove to be useful for future benchmarking purposes. The stacking sequence of the panel is presented in Fig. 16, while the materials are those reported in Table 6. Note that the ply angles are no longer restricted to 0 and 90 , but a more realistic stacking sequence is now considered that includes plies at \pm 45 . Two distinct loading conditions are assumed, as sketched in Fig. 16: a bisinusoidal and a uniformly distributed pressure, applied at the top of the plate.

The results are presented by considering four different sublaminate models of increasing complexity. The first one, FSDT/ED E32 $_{32}$ FSDT, has 15 T-DOF and employs a FSDT description of the inner and the outer shell, while the two-layer core is represented with an equivalent single layer approach. To highlight the potential benefits of an improved core description, the second model is chosen to be FSDT/LD L $_{32} / \mathrm{FSDT}$, in which a layerwise approach is adopted for the core, while the facesheets are still modeled referring to FSDT. The third set of results is computed by refining the description of the inner shell, i.e., the thicker one, by replacing the FSDT model with a $\mathrm{ED}_{332}$ theory. Finally, in the fourth configuration both the inner and the outer shell are modeled using $\mathrm{ED}_{332}$ theory. The results obtained with this latter model, although not exact, are here taken as reference. The number of T-DOF of the latter three models are 23, 29 and 35, respectively. 


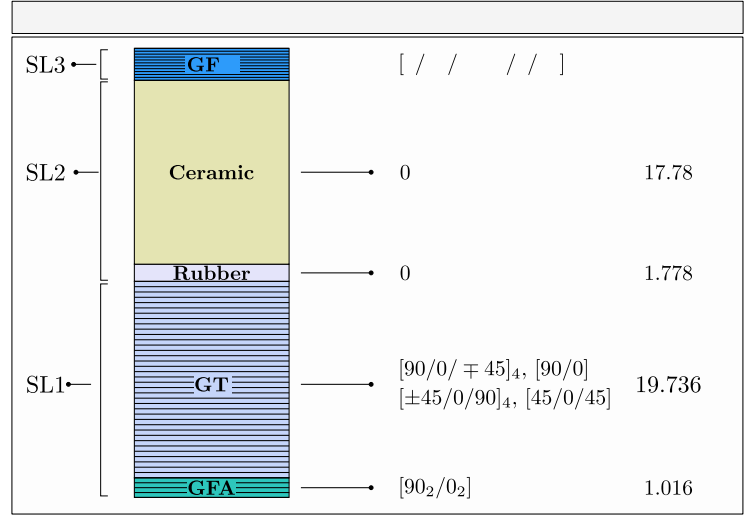

(a)

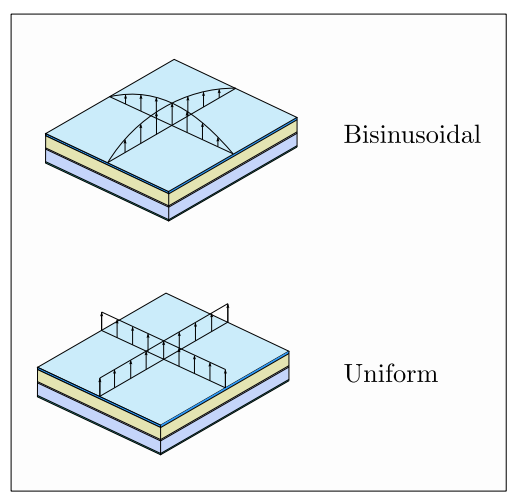

(b)

Fig. 16. Sandwich panel S4: (a) stacking sequence and sublaminate representation, (b) loading conditions.

Table 8

Panel S4 subjected to bisinusoidal pressure.

\begin{tabular}{|c|c|c|c|c|c|c|c|c|c|c|}
\hline \multirow[t]{3}{*}{ Theory } & & \multicolumn{9}{|c|}{ Boundary conditions } \\
\hline & & \multicolumn{3}{|l|}{ SSSS } & \multicolumn{3}{|l|}{ SCSC } & \multicolumn{3}{|l|}{$\mathrm{CCCC}$} \\
\hline & & $u_{z}(\mathrm{~mm})$ & $\sigma_{x x} / q$ & $\sigma_{y y} / q$ & $u_{z}(\mathrm{~mm})$ & $\sigma_{x x} / q$ & $\sigma_{y y} / q$ & $u_{z}(\mathrm{~mm})$ & $\sigma_{x x} / q$ & $\sigma_{y y} / q$ \\
\hline \multirow[t]{3}{*}{$\mathrm{FSDT} / \mathrm{ED}_{332} / \mathrm{FSDT}$} & SL3 (top) & 0.1684 & 3.3805 & 3.3790 & 0.1291 & 2.7376 & 2.6474 & 0.1069 & 2.2402 & 2.2406 \\
\hline & SL1 (top) & 0.1453 & 15.9007 & 3.1813 & 0.1057 & 11.3650 & 2.5381 & 0.0833 & 9.9997 & 2.0219 \\
\hline & SL1 (bot) & & -9.4014 & -9.4632 & & -6.8882 & -7.4708 & & -5.7842 & -5.8800 \\
\hline \multirow[t]{3}{*}{ FSDT/LD $332 / F S D T$} & SL3 (top) & 0.1975 & 4.6254 & 4.6252 & 0.1504 & 3.6555 & 3.6458 & 0.1254 & 3.0540 & 3.0597 \\
\hline & SL1 (top) & 0.1599 & 18.8871 & 3.7877 & 0.1120 & 13.0617 & 2.9262 & 0.0866 & 11.2964 & 2.2889 \\
\hline & SL1 (bot) & & -10.1896 & -10.2340 & & -7.1706 & -8.0346 & & -6.1001 & -6.1967 \\
\hline \multirow[t]{3}{*}{$\mathrm{ED}_{332} / \mathrm{LD}_{332} / \mathrm{FSDT}$} & SL3 (top) & 0.1960 & 4.5906 & 4.5904 & 0.1494 & 3.6298 & 3.6177 & 0.1246 & 3.0327 & 3.0351 \\
\hline & SL1 (top) & 0.1584 & 19.7585 & 4.3221 & 0.1110 & 13.7348 & 3.5163 & 0.0858 & 12.3554 & 2.8573 \\
\hline & SL1 (bot) & 0.1574 & -10.4511 & -10.4792 & 0.1099 & -7.4304 & -8.4450 & 0.0847 & -6.4779 & -6.5414 \\
\hline \multirow[t]{3}{*}{$\mathrm{ED}_{332} / \mathrm{LD}_{332} / \mathrm{ED}_{332}$} & SL3 (top) & 0.1962 & 4.7535 & 4.7533 & 0.1496 & 3.7841 & 3.8045 & 0.1249 & 3.2164 & 3.2188 \\
\hline & SL1 (top) & 0.1583 & 19.7462 & 4.3194 & 0.1109 & 13.7260 & 3.5142 & 0.0857 & 12.3491 & 2.8558 \\
\hline & SL1 (bot) & 0.1573 & -10.4444 & -10.4725 & 0.1098 & -7.4254 & -8.4402 & 0.0846 & -6.4745 & -6.5380 \\
\hline
\end{tabular}

Table 9

Panel S4 subjected to uniformly distributed pressure.

\begin{tabular}{|c|c|c|c|c|c|c|c|c|c|c|}
\hline \multirow[t]{3}{*}{ Theory } & & \multicolumn{9}{|c|}{ Boundary conditions } \\
\hline & & \multicolumn{3}{|l|}{ SSSS } & \multicolumn{3}{|l|}{ SCSC } & \multicolumn{3}{|l|}{$\mathrm{CCCC}$} \\
\hline & & $u_{z}(\mathrm{~mm})$ & $\sigma_{x x} / q$ & $\sigma_{y y} / q$ & $u_{z}(\mathrm{~mm})$ & $\sigma_{x x} / q$ & $\sigma_{y y} / q$ & $u_{z}(\mathrm{~mm})$ & $\sigma_{x x} / q$ & $\sigma_{y y} / q$ \\
\hline \multirow[t]{3}{*}{$\mathrm{FSDT} / \mathrm{ED}_{332} / \mathrm{FSDT}$} & SL3 (top) & 0.2474 & 4.0666 & 4.0587 & 0.1797 & 2.9530 & 2.8201 & 0.1417 & 2.1264 & 2.1236 \\
\hline & SL1 (top) & 0.2248 & 22.8195 & 4.5491 & 0.1565 & 15.0284 & 3.4502 & 0.1181 & 12.7588 & 2.5732 \\
\hline & SL1 (bot) & & -13.5526 & -13.5938 & & -9.2324 & -10.1966 & & -7.3887 & -7.4914 \\
\hline \multirow[t]{3}{*}{$\mathrm{FSDT} / \mathrm{LD}_{332} / \mathrm{FSDT}$} & SL3 (top) & 0.2857 & 5.4657 & 5.4562 & 0.2050 & 3.7981 & 3.7867 & 0.1624 & 2.7852 & 2.7882 \\
\hline & SL1 (top) & 0.2487 & 27.5404 & 5.5051 & 0.1666 & 17.5677 & 4.0408 & 0.1234 & 14.6625 & 2.9634 \\
\hline & SL1 (bot) & & -14.8438 & -14.8545 & & -9.6834 & -11.1078 & & -7.8966 & -7.9998 \\
\hline \multirow[t]{3}{*}{$\mathrm{ED}_{332} / \mathrm{LD}_{332} / \mathrm{FSDT}$} & SL3 (top) & 0.2833 & 5.4101 & 5.3987 & 0.2033 & 3.7589 & 3.7415 & 0.1612 & 2.7539 & 2.7499 \\
\hline & SL1 (top) & 0.2463 & 28.4188 & 6.0458 & 0.1649 & 18.1087 & 4.6803 & 0.1221 & 15.8877 & 3.5689 \\
\hline & SL1 (bot) & 0.2453 & -15.0150 & -14.9969 & 0.1639 & -9.8610 & -11.5261 & 0.1210 & -8.2630 & -8.3124 \\
\hline \multirow[t]{3}{*}{$\mathrm{ED}_{332} / \mathrm{LD}_{332} / \mathrm{ED}_{332}$} & SL3 (top) & 0.2833 & 5.5735 & 5.5620 & 0.2035 & 3.9049 & 3.9565 & 0.1614 & 2.9646 & 2.9606 \\
\hline & SL1 (top) & 0.2461 & 28.4039 & 6.0427 & 0.1648 & 18.0996 & 4.6783 & 0.1220 & 15.8838 & 3.5681 \\
\hline & SL1 (bot) & 0.2451 & -15.0070 & -14.9889 & 0.1637 & -9.8554 & -11.5222 & 0.1210 & -8.2616 & -8.3111 \\
\hline
\end{tabular}

Numerical results are reported in Tables 8 and 9 for the bisinusoidal and the uniform loading, respectively. In order to ensure the convergence up to the third digit, results are calculated using $60 \times 60$ Ritz functions. Three sets of boundary conditions are considered, namely simply-supported panel (SSSS), fully clamped (CCCC), and an intermediate condition with two parallel sides simply-supported and the other two clamped (SCSC). The trans- verse displacement $u_{z}$ and the stresses $\sigma_{x x}$ and $\sigma_{y y}$ are reported at the center of the panel, at three locations along the thickness direction: the top of the outer shell, and the top and bottom of the inner shell.

As seen from Tables 8 and 9, the predictions obtained using FSDT/ED B $_{32} /$ FSDT display a noticeable discrepancy with the results obtained with the more refined models. The stress components at 


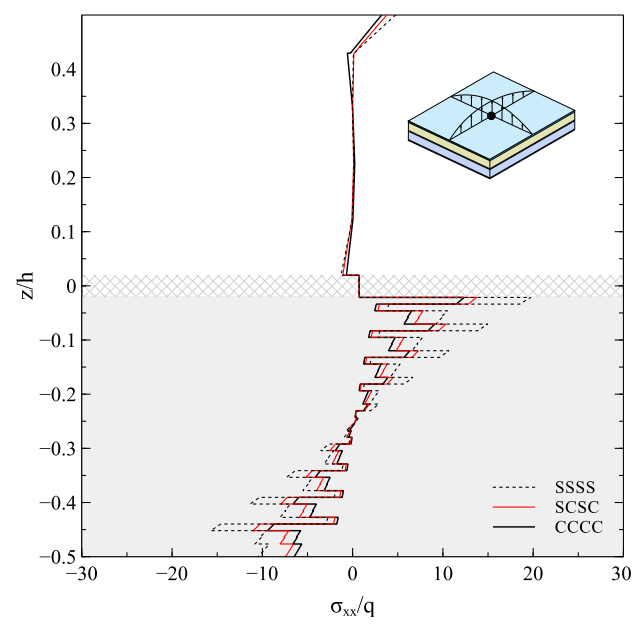

(a)

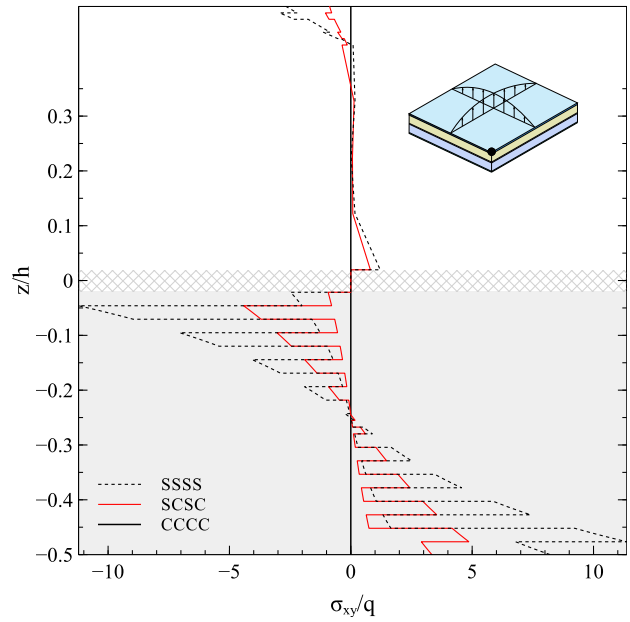

(b)

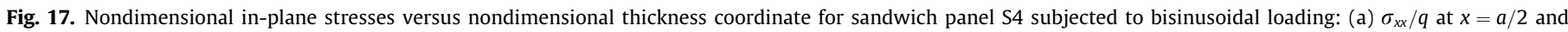
$y=b / 2$, (b) $\sigma_{x y} / q$ at $x=0$ and $y=0$.

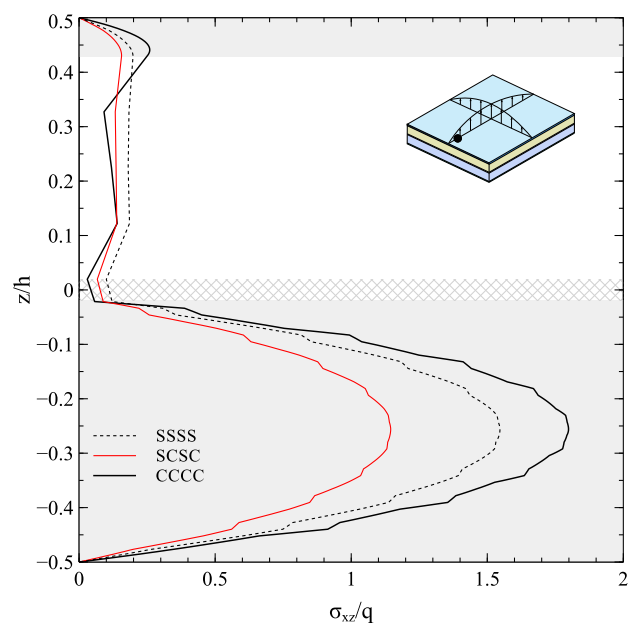

(a)

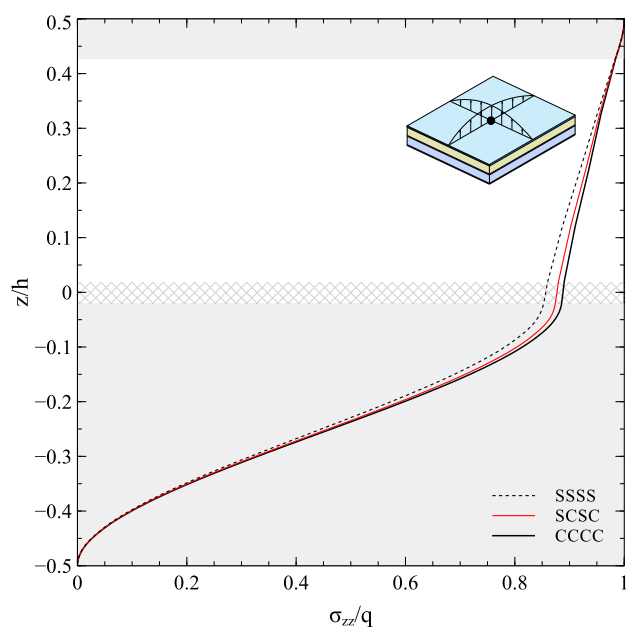

(b)

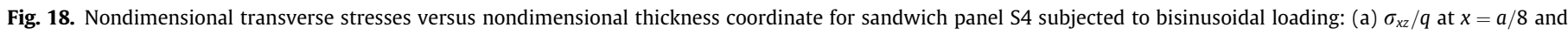
$y=b / 2$, (b) $\sigma_{z z} / q$ at $x=a / 2$ and $y=b / 2$.

the outer shell (SL3) are underestimated by more than $25 \%$ in all the cases, and even the out of plane displacement, which is in fact a global quantity, differs of more than $10 \%$ with respect to the reference solution. It is clear that the composite core, made out of two materials with a drastic variation of the elastic properties along the $z$ direction, calls for a layerwise description.

Adopting the FSDT/LD ${ }_{332} /$ FSDT theory provides a significant improvement of the quality of the results, thus demonstrating the need for a properly refined core modeling. The results can be considered satisfactory especially for the third sublaminate, SL3, representing the outer shell. It can be observed that the transverse displacements are predicted with a maximum difference that is below $1 \%$ with respect to the reference solution. On the contrary, the prediction of the stresses is less accurate, especially in correspondence of the inner shell (SL1), where the differences can be higher than 10\%. As seen from Fig. 16, the inner shell is approximately seven times thicker than the outer shell. It follows that
FSDT can be appropriate for modeling SL3, while the adoption of a higher-order theory is beneficial to improve the stress prediction inside SL1. As expected, the trend of the results is similar for the bisinusoidal and the uniform load.

The plots of the through-the-thickness stress distributions for the bisinusoidal loading condition is provided in Figs. 17 and 18. The results are obtained with the $\mathrm{ED}_{332} / \mathrm{LD}_{332} / \mathrm{FSDT}$ theory. The normalized in-plane stresses are reported in Fig. 17. Fig. 17 shows that the intensity of the bending stresses increases as the constraints are removed from the panel. The fully clamped panel is the one associated with the lower magnitude of bending deflections, thus the stress level is the smallest among the three configurations. On the contrary, the SSSS panel is the one undergoing the highest amount of in-plane stress $\sigma_{x x}$. Fig. 17 reports the distribution of the in-plane shear stress $\sigma_{x y}$ evaluated at one of the four corners. The shear stress is obviously nil in the CCCC case, whereas it is of the same order of magnitude of the bending stress 
for the SSSS and the SCSC configurations. The role played by the boundary conditions is thus relevant and the magnitude of the stresses changes in the three cases here examined.

The normalized transverse stress response in presented in Fig. 18. Fig. 18 reveals a similar trend of the transverse shear stress $\sigma_{x z}$ for the three cases, where the CCCC panel appears to build the highest stress level. The normal stress components $\sigma_{z z}$ is finally reported in Fig. 18, where the response can be seen as almost insensitive to the boundary conditions.

\section{Conclusions}

The paper has illustrated the development of a numerical tool, here denoted as S-GUF-Ritz, for the bending analysis of multilayered structures. The method combines the idealization of the structure based on the S-GUF theory and the method of Ritz for the approximate solution of the problem.

Main advantage of the proposed approach is the possibility of combining any sort of axiomatic theories, ELS or LW, while dividing the plate into an arbitrary set of sublaminates. This strategy has the effect of providing great flexibility of analysis, and can be exploited to refine the kinematic description only in those regions where refinement is effectively needed. As a consequence, the number of degrees of freedom can be kept at minimum, while guaranteeing high fidelity of the results. Despite the generality of the approach, the theoretical formulation is achieved in a simple manner, and relies on the expansion and assembly of theoryindependent expression, denoted kernels.

Sandwich panels are typical configurations that can benefit from the capabilities offered by the S-GUF-Ritz approach, where the two facesheets and the core can be modeled with three distinct sublaminates. However, any other combination can be defined, without any sort of restriction. The importance of allowing the choice between different theories has been demonstrated in the examples discussed in the paper. For instance, the sandwich panels S1 and S3, whose core is made of one single material, are properly modeled with a FSDT/ED ${ }_{332} /$ FSDT model. On the other hand, the configurations S2 and S4, where the core is composed of two distinct materials, demand the adoption of a layerwise core modeling. At least, a FSDT/LD L $_{32} /$ FSDT model is needed in these cases. As discussed in the paper, the quality of the predictions obtained by means of a sublaminate modeling is much higher in comparison to purely ESL models with comparable number of degrees of freedom. Thanks to the efficient implementation of the method, a relatively high number of shape functions can be considered, and refined results can be obtained both in terms of global and local quantities, such as the stress field arising from localized loads. The comparison with 3D exact solutions from the literature reveals very close matching with S-GUF-Ritz results, both in terms of in-plane and transverse stress components. In addition, the Ritz solution offers the possibility of accounting for any combination of boundary conditions along the four panel edges, and no restrictions are introduced with regard to stacking sequences. The S-GUF-Ritz method is then an interesting method to perform the stress analysis of a wide range of configurations that go beyond the academic benchmark ones and for which no exact elasticity solution is available.

The effectiveness of the proposed approach suggests its extension to other kinds of solution procedures, such as buckling, free vibration, dynamic transient analyses. These topics are being currently investigated and are the subjects of ongoing research activities.

\section{Appendix A. Appendix}

\section{A.1. Principle of Virtual Displacements}

$$
\begin{aligned}
& \sum_{k=1}^{N_{k}} \sum_{p=1}^{N_{p}^{k}}\left[\delta u_{x \alpha_{u_{x} i} i}^{p, k} \widetilde{C}_{11}^{p, k} Z_{u_{x} u_{x}}^{p \alpha_{u_{x}} \beta_{u_{x}}} \mathcal{I}_{u_{x} u_{x} j}^{1010} u_{x \beta_{u_{x} j} j}^{p, k}+\delta u_{x u_{u_{x} i} i}^{p, k} \widetilde{C}_{12}^{p, k} Z_{u_{x} u_{y}}^{p \alpha_{u_{x}} \beta_{u_{y}}} \mathcal{I}_{u_{x} u_{y} i j}^{1001} u_{y \beta_{u_{y}} j}^{p, k}\right. \\
& +\delta u_{x u_{u_{x} i}}^{p, k} \widetilde{C}_{16}^{p, k} Z_{u_{x} u_{x}}^{p \alpha_{u_{x}} \beta_{u_{x}}} \mathcal{I}_{u_{x} u_{x} j}^{1001} u_{x \beta_{u_{x} j} j}^{p, k}+\delta u_{x \alpha_{u_{x} i}}^{p, k} \widetilde{C}_{16}^{p, k} Z_{u_{x} u_{y}}^{p \alpha_{u_{x}} \beta_{u_{y}}} \mathcal{I}_{u_{x} u_{y} i j}^{1010} u_{y \beta_{u_{y}} j}^{p, k} \\
& +\delta u_{y \alpha_{u y} i}^{p, k} \widetilde{C}_{12}^{p, k} Z_{u_{y} u_{x}}^{p \alpha_{u_{y}} \beta_{u_{x}}} \mathcal{I}_{u_{y} u_{x} i j}^{0110} u_{x \beta_{u_{x} j} j}^{p, k}+\delta u_{y \alpha_{u_{y} i}}^{p, k} \widetilde{C}_{22}^{p, k} Z_{u_{y} u_{y}}^{p \alpha_{u_{y}} \beta_{u_{y}}} \mathcal{I}_{u_{y} u_{y} i j}^{0101} u_{y \beta_{u_{y}} j}^{p, k} \\
& +\delta u_{y \alpha_{u y} i}^{p, k} \widetilde{C}_{26}^{p, k} Z_{u_{y} u_{x}}^{p \alpha_{u_{y}} \beta_{u_{x}}} \mathcal{I}_{u_{y} u_{x} i j}^{0101} u_{x \beta_{u_{x} j} j}^{p, k}+\delta u_{y \alpha_{u y} i}^{p, k} \widetilde{C}_{26}^{p, k} Z_{u_{y} u_{y}}^{p \alpha_{u_{y}} \beta_{u_{y}}} \mathcal{I}_{u_{y} u_{y} i j}^{0110} u_{y \beta_{u y} j}^{p, k} \\
& +\delta u_{x \alpha_{u_{x}}}^{p, k} \widetilde{C}_{16}^{p, k} Z_{u_{x} u_{x}}^{p \alpha_{u_{x}} \beta_{u_{x}}} \mathcal{I}_{u_{x} u_{x} i j}^{0110} u_{x \beta_{u_{x}} j}^{p, k}+\delta u_{x \alpha_{u_{x} i}}^{p, k} \widetilde{C}_{26}^{p, k} Z_{u_{x} u_{y}}^{p \alpha_{u_{x}} \beta_{u_{y}}} \mathcal{I}_{u_{x} u_{y} j}^{0101} u_{y \beta_{u_{y} j} j}^{p, k} \\
& +\delta u_{x \alpha_{u_{x} i} i}^{p, k} \widetilde{C}_{66}^{p, k} Z_{u_{x} u_{x}}^{p \alpha_{u_{x}} \beta_{u_{x}}} \mathcal{I}_{u_{x} u_{x} j}^{0101} u_{x \beta_{u_{x} j} j}^{p, k}+\delta u_{x \alpha_{u_{x} i}}^{p, k} \widetilde{C}_{66}^{p, k} Z_{u_{x} u_{y}}^{p \alpha_{u_{x}} \beta_{u_{y}}} \mathcal{I}_{u_{x} u_{y} i j}^{0110} u_{y \beta_{u_{y} j} j}^{p, k} \\
& +\delta u_{y \alpha_{u_{y} i} i}^{p, k} \widetilde{C}_{16}^{p, k} Z_{u_{y} u_{x}}^{p \alpha_{u_{y}} \beta_{u_{x}}} \mathcal{I}_{u_{y} u_{x} i j}^{1010} u_{x \beta_{u_{x} j} j}^{p, k}+\delta u_{y \alpha_{u_{y} i} i}^{p, k} \widetilde{C}_{26}^{p, k} Z_{u_{y} u_{y}}^{p \alpha_{u_{y}} \beta_{u_{y}}} \mathcal{I}_{u_{y} u_{y} i j}^{1001} u_{y \beta_{u_{y}} j}^{p, k} \\
& +\delta u_{y \alpha_{u y} i}^{p, k} \widetilde{C}_{66}^{p, k} Z_{u_{y} u_{x}}^{p \alpha_{u_{u_{x}} \beta_{u_{x}}}} \mathcal{I}_{u_{y} u_{x} i j}^{1001} u_{x \beta_{u_{x} j} j}^{p, k}+\delta u_{y \alpha_{u_{y} i} i}^{p, k} \widetilde{C}_{66}^{p, k} Z_{u_{y} u_{y}}^{p \alpha_{u_{y}} \beta_{u_{y}}} \mathcal{I}_{u_{y} u_{y} i j}^{1010} u_{y \beta_{u_{y} j} j}^{p, k} \\
& +\delta u_{z \alpha_{u_{z} i} i}^{p, k} \widetilde{C}_{55}^{p, k} Z_{u_{z} u_{z}}^{p \alpha_{u_{z}} \beta_{u_{z}}} \mathcal{I}_{u_{z} u_{z} i j}^{1010} u_{z \beta_{u_{z} j} j}^{p, k}+\delta u_{z \alpha_{u_{z} i} i}^{p, k} \widetilde{C}_{55}^{p, k} Z_{u_{z} \partial u_{x}}^{p \alpha_{u_{z}} \beta_{u_{x}}} \mathcal{I}_{u_{z} u_{x} i j}^{1000} u_{x \beta_{u_{x}} j}^{p, k} \\
& +\delta u_{z \alpha_{u_{z}} i}^{p, k} \widetilde{C}_{45}^{p, k} Z_{u_{z} u_{z}}^{p \alpha_{u_{z}} \beta_{u_{z}}} \mathcal{I}_{u_{z} u_{z} i j}^{1001} u_{z \beta_{u_{z}} j}^{p, k}+\delta u_{z u_{u_{z} i} i}^{p, k} \widetilde{C}_{45}^{p, k} Z_{u_{z} \partial u_{y}}^{p \alpha_{u_{z}} \beta_{u_{y}}} \mathcal{I}_{u_{z} u_{y} j j}^{1000} u_{y \beta_{u_{y}} j}^{p, k} \\
& +\delta u_{z \alpha_{u_{z} i} i}^{p, k} \widetilde{C}_{45}^{p, k} Z_{u_{z} u_{z}}^{p \alpha_{u_{z}} \beta_{u_{z}}} \mathcal{I}_{u_{z} u_{z} i j}^{0110} u_{z \beta_{u_{z} j} j}^{p, k}+\delta u_{z \alpha_{u_{z} i}}^{p, k} \widetilde{C}_{45}^{p, k} Z_{u_{z} \partial u_{x}}^{p \alpha_{u_{z}} \beta_{u_{x}}} \mathcal{I}_{u_{z} u_{x} i j}^{0100} u_{x \beta_{u_{x} j} j}^{p, k} \\
& +\delta u_{z \alpha_{u_{z}} i}^{p, k} \widetilde{C}_{44}^{p, k} Z_{u_{z} u_{z}}^{p \alpha_{u_{z}} \beta_{u_{z}}} \mathcal{I}_{u_{z} u_{z} i j}^{0101} u_{z \beta_{u_{z}} j}^{p, k}+\delta u_{z \alpha_{u_{z} i}}^{p, k} \widetilde{C}_{44}^{p, k} Z_{u_{z} \partial u_{y}}^{p \alpha_{u_{z}} \beta_{u_{y}}} \mathcal{I}_{u_{z} u_{y} j j}^{0100} u_{y \beta_{u_{y} j} j}^{p, k} \\
& +\delta u_{x \alpha_{u_{x} i} i}^{p, k} \widetilde{C}_{55}^{p, k} Z_{\partial u_{x} u_{z}}^{p \alpha_{u_{x}} \beta_{u_{z}}} \mathcal{I}_{u_{x} u_{z} i j}^{0010} u_{z \beta_{u_{z} j} j}^{p, k}+\delta u_{x \alpha_{u_{x} i}}^{p, k} \widetilde{C}_{55}^{p, k} Z_{\partial u_{x} \partial u_{x}}^{p \alpha_{u_{x}} \beta_{u_{x}}} \mathcal{I}_{u_{x} u_{x} i j}^{0000} u_{x \beta_{u_{x}} j}^{p, k} \\
& +\delta u_{x u_{u_{x} i} i}^{p, k} \widetilde{C}_{45}^{p, k} Z_{\partial u_{x} u_{z}}^{p \alpha_{u_{z}} \beta_{u_{z}}} \mathcal{I}_{u_{x} u_{z} i j}^{0001} u_{z \beta_{u_{z} j} j}^{p, k}+\delta u_{x u_{u_{x} i} i}^{p, k} \widetilde{C}_{45}^{p, k} Z_{\partial u_{x} \partial u_{y}}^{p \alpha_{u_{x}} \beta_{u_{y}}} \mathcal{I}_{u_{x} u_{y} i j}^{0000} u_{y \beta_{u_{y}} j}^{p, k} \\
& +\delta u_{y \alpha_{u_{y} i} i}^{p, k} \widetilde{C}_{45}^{p, k} Z_{\partial u_{y} u_{z}}^{p \alpha_{u_{y}} \beta_{u_{z}}} \mathcal{I}_{u_{y} u_{z} i j}^{0010} u_{z \beta_{u_{z} j} j}^{p, k}+\delta u_{y \alpha_{u_{y} i}}^{p, k} \widetilde{C}_{45}^{p, k} Z_{\partial u_{y} \partial u_{x}}^{p \alpha_{u_{y}} \beta_{u_{x}}} \mathcal{I}_{u_{y} u_{x} i j}^{0000} u_{x \beta_{u_{x} j} j}^{p, k} \\
& +\delta u_{y \alpha_{u_{y} i} i}^{p, k} \widetilde{C}_{44}^{p, k} Z_{\partial u_{y} u_{z}}^{p \alpha_{u_{y}} \beta_{u_{z}}} \mathcal{I}_{u_{y} u_{z} i j}^{0001} u_{z \beta_{u_{z} j} j}^{p, k}+\delta u_{y \alpha_{u_{y} i}}^{p, k} \widetilde{C}_{44}^{p, k} Z_{\partial u_{y} \partial u_{y}}^{p \alpha_{u_{y}} \beta_{u_{y}}} \mathcal{I}_{u_{y} u_{y} i j}^{0000} u_{y \beta_{u y} j}^{p, k} \\
& +\delta u_{x \alpha_{u_{x} i} i}^{p, k} \widetilde{C}_{13}^{p, k} Z_{u_{x} \partial u_{z}}^{p \alpha_{u_{x}} \beta_{u_{z}}} \mathcal{I}_{u_{x} u_{z} i j}^{1000} u_{z \beta_{u_{z} j} j}^{p, k}+\delta u_{y \alpha_{u_{y} i}}^{p, k} \widetilde{C}_{23}^{p, k} Z_{u_{y} \partial u_{z}}^{p \alpha_{u_{y}} \beta_{u_{z}}} \mathcal{I}_{u_{y} u_{z} i j}^{0100} u_{z \beta_{u_{z}} j}^{p, k} \\
& +\delta u_{x \alpha_{u_{x} i} i}^{p, k} \widetilde{C}_{36}^{p, k} Z_{u_{x} \partial u_{z}}^{p \alpha_{u_{x}} \beta_{u_{z}}} \mathcal{I}_{u_{x} u_{z} i j}^{0100} u_{z \beta_{u_{z} j} j}^{p, k}+\delta u_{y \alpha_{u_{y} i} i}^{p, k} \widetilde{C}_{36}^{p, k} Z_{u_{y} \partial u_{z}}^{p \alpha_{u_{y}} \beta_{u_{z}}} \mathcal{I}_{u_{y} u_{z} i j}^{1000} u_{z \beta_{u_{z}} j}^{p, k} \\
& +\delta u_{z \alpha_{u_{z}} i}^{p, k} \widetilde{C}_{13}^{p, k} Z_{\partial u_{z} u_{x}}^{p \alpha_{u_{z}} \beta_{u_{x}}} \mathcal{I}_{u_{z} u_{x} i j}^{0010} u_{x \beta_{u_{x} j} j}^{p, k}+\delta u_{z \alpha_{u_{z} i}}^{p, k} \widetilde{C}_{23}^{p, k} Z_{\partial u_{z} u_{y}}^{p \alpha_{u_{z}} \beta_{u_{y}}} \mathcal{I}_{u_{z} u_{y} i j}^{0001} u_{y \beta_{u y} j}^{p, k} \\
& +\delta u_{z \alpha_{u_{z}} i}^{p, k} \widetilde{C}_{36}^{p, k} Z_{\partial u_{z} u_{x}}^{p \alpha_{u_{z}} \beta_{u_{x}}} \mathcal{I}_{u_{z} u_{x} i j}^{0001} u_{x \beta_{u_{x} j} j}^{p, k}+\delta u_{z \alpha_{u_{z} i}}^{p, k} \widetilde{C}_{36}^{p, k} Z_{\partial u_{z} u_{y}}^{p \alpha_{u_{z}} \beta_{u_{y}}} \mathcal{I}_{u_{z} u_{y} i j}^{0010} u_{y \beta_{u y} j}^{p, k} \\
& \left.+\delta u_{z \alpha_{u_{z}} i}^{p, k} \widetilde{C}_{33}^{p, k} Z_{\partial u_{z} \partial u_{z}}^{p \alpha_{u_{z}} \beta_{u_{z}}} \mathcal{I}_{u_{z} u_{z} j}^{0000} u_{z \beta_{u_{z}} j}^{p, k}\right] \\
& =\delta u_{z 0 i}^{N_{p}^{k}, N_{k}} \int_{\Omega} N_{u_{z}} f_{z}^{\text {top }} \mathrm{d} \Omega+\delta u_{z 1 i}^{1,1} \int_{\Omega} N_{u_{z}} f_{z}^{\text {bot }} \mathrm{d} \Omega
\end{aligned}
$$

\section{A.2. Stiffness matrix}

Define the matrices $\mathcal{I}_{u_{r} u_{s}}^{\text {defg }}$ such that:

$\left(\mathcal{I}_{u_{r} u_{s}}^{\text {defg }}\right)_{i j}=\mathcal{I}_{u_{r} u_{s} i j}^{\text {defg }}$

where $\mathcal{I}_{u_{r} u_{i j}}^{\text {defg }}$ is defined by Eq. (30). The sub-blocks composing the stiffness matrix of Eq. (41) are: 


$$
\begin{aligned}
\mathbf{K}_{u_{x} u_{x}}= & \mathcal{I}_{u_{x} u_{x}}^{1010} \otimes \mathbf{Z}_{u_{x} u_{x} 11}+\left(\mathcal{I}_{u_{x} u_{x}}^{1001}+\mathcal{I}_{u_{x} u_{x}}^{0110}\right) \otimes \mathbf{Z}_{u_{x} u_{x} 16}+\mathcal{I}_{u_{x} u_{x}}^{0101} \otimes \mathbf{Z}_{u_{x} u_{x} 66} \\
& +\mathcal{I}_{u_{x} u_{x}}^{0000} \otimes \mathbf{Z}_{\partial u_{x} \partial u_{x} 55} \\
\mathbf{K}_{u_{x} u_{y}}= & \mathcal{I}_{u_{x} u_{y}}^{1001} \otimes \mathbf{Z}_{u_{x} u_{y} 12}+\mathcal{I}_{u_{x} u_{y}}^{1010} \otimes \mathbf{Z}_{u_{x} u_{y} 16}+\mathcal{I}_{u_{x} u_{y}}^{0101} \otimes \mathbf{Z}_{u_{x} u_{y} 26} \\
& +\mathcal{I}_{u_{x} u_{y}}^{0110} \otimes \mathbf{Z}_{u_{x} u_{y} 66}+\mathcal{I}_{u_{x} u_{y}}^{0000} \otimes \mathbf{Z}_{\partial u_{x} \partial u_{y} 45} \\
\mathbf{K}_{u_{x} u_{z}}= & \mathcal{I}_{u_{x} u_{z}}^{0010} \otimes \mathbf{Z}_{\partial u_{x} u_{z} 55}+\mathcal{I}_{u_{x} u_{z}}^{0001} \otimes \mathbf{Z}_{\partial u_{x} u_{z} 45}+\mathcal{I}_{u_{x} u_{z}}^{1000} \otimes \mathbf{Z}_{u_{x} \partial u_{z} 13} \\
& +\mathcal{I}_{u_{x} u_{z}}^{0100} \otimes \mathbf{Z}_{u_{x} \partial u_{z} 36} \\
\mathbf{K}_{u_{y} u_{y}}= & \mathcal{I}_{u_{y} u_{y}}^{0101} \otimes \mathbf{Z}_{u_{y} u_{y} 22}+\left(\mathcal{I}_{u_{y} u_{y}}^{0110}+\mathcal{I}_{u_{y} u_{y}}^{1001}\right) \otimes \mathbf{Z}_{u_{y} u_{y} 26}+\mathcal{I}_{u_{y} u_{y}}^{1010} \otimes \mathbf{Z}_{u_{y} u_{y} 66} \\
& +\mathcal{I}_{u_{y} u_{y}}^{0000} \otimes \mathbf{Z}_{\partial u_{y} \partial u_{y} 44} \\
\mathbf{K}_{u_{y} u_{z}}= & \mathcal{I}_{u_{y} u_{z}}^{0010} \otimes \mathbf{Z}_{\partial u_{y} u_{z} 45}+\mathcal{I}_{u_{y} u_{z}}^{0001} \otimes \mathbf{Z}_{\partial u_{y} u_{z} 44}+\mathcal{I}_{u_{y} u_{z}}^{0100} \otimes \mathbf{Z}_{u_{y} \partial u_{z} 23} \\
& +\mathcal{I}_{u_{y} u_{z}}^{1000} \otimes \mathbf{Z}_{u_{y} \partial u_{z} 36} \\
\mathbf{K}_{u_{z} u_{z}}= & \mathcal{I}_{u_{z} u_{z}}^{1010} \otimes \mathbf{Z}_{u_{z} u_{z} 55}+\left(\mathcal{I}_{u_{z} u_{z}}^{1001}+\mathcal{I}_{u_{z} u_{z}}^{0110}\right) \otimes \mathbf{Z}_{u_{z} u_{z} 45}+\mathcal{I}_{u_{z} u_{z}}^{0101} \otimes \mathbf{Z}_{u_{z} u_{z} 44} \\
& +\mathcal{I}_{u_{z} u_{z}}^{0000} \otimes \mathbf{Z}_{\partial u_{z} \partial u_{z} 33}
\end{aligned}
$$

where the symbol $\otimes$ denotes the Kronecker product, which is defined as:

$\mathbf{A} \otimes \mathbf{B}=\left[\begin{array}{ccc}A_{11} \mathbf{B} & \ldots & A_{1 N} \mathbf{B} \\ \vdots & & \vdots \\ A_{M 1} \mathbf{B} & \ldots & A_{M N} \mathbf{B}\end{array}\right]$

Note that the matrix resulting from the product $\mathbf{A} \otimes \mathbf{B}$ has dimension $(M \times P) \times(N \times Q)$ if $M \times N$ and $P \times Q$ are the dimensions of the matrices $\mathbf{A}$ and $\mathbf{B}$, respectively.

\section{A.3. Vector of loads}

Define the vectors $\mathcal{I}_{u_{z} f_{z}}^{\text {bot }}$ and $\mathcal{I}_{u_{z} f_{z}}^{\text {top }}$ such that:

$\left(\mathcal{I}_{u_{z} f_{z}}^{\text {top }}\right)_{i}=\mathcal{I}_{u_{z} f_{z} i}^{\text {top }} \quad\left(\mathcal{I}_{u_{z} f_{z}}^{\text {bot }}\right)_{i}=\mathcal{I}_{u_{z} f_{z} i}^{\text {bot }}$

where $\mathcal{I}_{u_{z} f_{z} i}^{\text {bot }}$ and $\mathcal{I}_{u_{z} f_{z} i}^{\text {top }}$ are defined by Eq. (28).

The vectors of the external loads are defined as:

$\mathbf{L}=\mathcal{I}_{u_{z_{z}}}^{\text {top }} \otimes \mathbf{L}_{i}^{\text {top }}+\mathcal{I}_{u_{z} f_{z}}^{\text {bot }} \otimes \mathbf{L}_{i}^{\text {bot }}$

with:

$\mathbf{L}_{i}^{\text {top }}=\left\{\begin{array}{lll}\mathbf{0} & \mathbf{0} & \mathbf{L}_{z i}^{\text {top }}\end{array}\right\}^{\mathrm{T}} \quad \mathbf{L}_{i}^{\text {bot }}=\left\{\begin{array}{lll}\mathbf{0} & \mathbf{0} & \mathbf{L}_{z i}^{\text {bot }}\end{array}\right\}^{\mathrm{T}}$

where the vectors $\mathbf{L}_{i}^{\text {top }}$ and $\mathbf{L}_{i}^{\text {bot }}$ are defined by Eq. (38).

\section{References}

[1] Reddy JN. Mechanics of laminated composite plates and shells: theory and analysis. Boca Raton: CRC Press; 2004.

[2] Carrera E. Historical review of zig-zag theories for multilayered plates and shells. Appl Mech Rev 2003:56(3):287-308.

[3] Frostig Y, Baruch M, Vilnay O, Sheinman I. High-order theory for sandwichbeam behavior with transversely flexible core. J Eng Mech 1992;118 (5):1026-43.

[4] Cho YB, Averill RC. An improved theory and finite-element model for laminated composite and sandwich beams using first-order zig-zag sublaminate approximations. Compos Struct 1997;37(3):281-98.

[5] Cho YB, Averill RC. First-order zig-zag sublaminate plate theory and finite element model for laminated composite and sandwich panels. Compos Struct 2000;50(1):1-15.

[6] Pantano A, Averill RC. A 3D zig-zag sublaminate model for analysis of thermal stresses in laminated composite and sandwich plates. J Sandwich Struct Mater 2000;2(3):288-312.

[7] Yip YC, Averill RC. A three-dimensional laminated plate finite element with high-order zig-zag sublaminate approximations. Int J Comput Eng Sci 2001;2 (1):137-80.

[8] Pai PF, Palazotto AN. A higher-order sandwich plate theory accounting for 3-D stresses. Int J Solids Struct 2001;38(30):5045-62.

[9] Pai PF, Palazotto AN. Two-dimensional sublamination theory for analysis of functionally graded plates. J Sound Vib 2007;308(1):164-89.
[10] Kärger L, Wetzel A, Rolfes R, Rohwer K. A three-layered sandwich element with improved transverse shear stiffness and stresses based on FSDT. Comput Struct 2006;84(13):843-54.

[11] Williams TO. A new, unified, theoretical framework for the formulation of general, nonlinear, single-scale shell theories. Compos Struct 2014; $107: 544-58$

[12] D'Ottavio M. A sublaminate generalized unified formulation for the analysis of composite structures. Compos Struct 2016;142:187-99.

[13] Carrera E. A class of two-dimensional theories for anisotropic multilayered plates analysis. Atti Accademia delle Scienze di Torino Mem Sci Fis $1995 ; 19: 1-39$.

[14] Carrera E. Theories and finite elements for multilayered, anisotropic, composite plates and shells. Arch Comput Methods Eng 2002;9(2):87-140.

[15] Carrera E, Demasi L. Classical and advanced multilayered plate elements based upon PVD and RMVT. Part 1: derivation of finite element matrices. Part 2: numerical implementations. Int J Numer Meth Eng 2002;55(2):191-231. 55 (3):253-291.

[16] Carrera E. Theories and finite elements for multilayered plates and shells: a unified compact formulation with numerical assessment and benchmarking. Arch Comput Methods Eng 2003;10(3):215-96.

[17] D’Ottavio M, Ballhause D, Wallmersperger T, Kröplin B. Considerations on higher-order finite elements for multilayered plates based on a unified formulation. Comput Struct 2006;84:1222-35.

[18] Carrera E, Cinefra M, Nali P. MITC technique extended to variable kinematic multilayered plate elements. Compos Struct 2010;92(8):1888-95.

[19] D’Ottavio M, Vidal P, Valot E, Polit O. Assessment of plate theories for free-edge effects. Compos Part B 2013;48:111-21.

[20] Ferreira AJM, Roque CMC, Carrera E, Cinefra M, Polit O. Two higher order zigzag theories for the accurate analysis of bending, vibration and buckling response of laminated plates by radial basis functions collocation and a unified formulation. J Compos Mater 2011;45(24):2523-36.

[21] Tornabene F, Fantuzzi N, Viola E, Carrera E. Static analysis of doubly-curved anisotropic shells and panels using CUF approach, differential geometry and differential quadrature method. Compos Struct 2014;107:675-97.

[22] Viola E, Tornabene F, Fantuzzi N. Stress and strain recovery of laminated composite doubly-curved shells and panels using higher-order formulations. Key Eng Mater, 624. p. 205-13.

[23] Dozio L, Carrera E. Ritz analysis of vibrating rectangular and skew multilayered plates based on advanced variable-kinematic models. Compos Struct 2012;94 (6):2118-28.

[24] Dozio L. Refined 2-D theories for free vibration analysis of annular plates: unified Ritz formulation and numerical assessment. Comput Struct 2015; $147: 250-8$.

[25] Vescovini R, Dozio L. A variable-kinematic model for variable stiffness plates: vibration and buckling analysis. Compos Struct 2016;142:15-26.

[26] Carrera E, Ciuffreda A. A unified formulation to assess theories of multilayered plates for various bending problems. Compos Struct 2005;69(3):271-93.

[27] Carrera E, Giunta G. Exact, hierarchical solutions for localized loadings in isotropic, laminated, and sandwich shells. J Pressure Vessel Technol 2009;131 (4):1-14.

[28] D'Ottavio M, Carrera E. Variable-kinematics approach for linearized buckling analysis of laminated plates and shells. AIAA J 2010;48(9):1987-96.

[29] Dozio L. Exact free vibration analysis of Lévy FGM plates with higher-order shear and normal deformation theories. Compos Struct 2014;111:415-25.

[30] Vescovini R, Dozio L. Exact refined buckling solutions for laminated plates under uniaxial and biaxial loads. Compos Struct 2015;127:356-68.

[31] Dozio L. A hierarchical formulation of the state-space Levy's method for vibration analysis of thin and thick multilayered shells. Compos Part B 2016;98:97-107.

[32] Demasi L. $\infty^{3}$ hierarchy plate theories for thick and thin composite plates: the generalized unified formulation. Compos Struct 2008;84(3):256-70.

[33] Demasi L. $\infty^{6}$ mixed plate theories based on the generalized unified formulation. Part I: governing equations. Compos Struct 2009;87(1):1-11.

[34] Demasi L. $\infty^{6}$ mixed plate theories based on the generalized unified formulation. Part V: results. Compos Struct 2009;88(1):1-16.

[35] Demasi L. Invariant finite element model for composite structures: the generalized unified formulation. AIAA J. 2010;48(8):1602-19.

[36] Demasi L. Partially zig-zag advanced higher order shear deformation theories based on the generalized unified formulation. Compos Struct 2012;94 (2):363-75.

[37] Demasi L. Partially layer wise advanced zig-zag and hsdt models based on the generalized unified formulation. Eng Struct 2013;53:63-91.

[38] Carrera E. A priori vs. a posteriori evaluation of transverse stresses in multilayered orthotropic plates. Compos Struct 2000;48(4):245-60.

[39] Barut A, Madenci E, Anderson T, Tessler A. Equivalent single-layer theory for a complete stress field in sandwich panels under arbitrarily distributed loading. Compos Struct 2002;58(4):483-95.

[40] Polit O, Touratier M. High-order triangular sandwich plate finite element for linear and non-linear analyses. Comput Methods Appl Mech Eng 2000; $185: 305-24$.

[41] Vel SS, Batra RC. Analytical solution for rectangular thick laminated plates subjected to arbitrary boundary conditions. AIAA J 1999;37(11):1464-73.

[42] Anderson T, Madenci E, Burton WS, Fish JC. Analytical solution of finitegeometry composite panels under transient surface loading. Int J Solids Struct 1998;35(12):1219-39. 